\title{
Quasinormal modes and holography
}

\author{
Pavel K. Kovtun \\ KITP, University of California, Santa Barbara, CA 93106-4030, USA \\ E-mail: kovtun@kitp.ucsb.edu \\ Andrei O. Starinets \\ Perimeter Institute for Theoretical Physics, Waterloo, ON N2L 2Y5, Canada \\ E-mail: starina@perimeterinstitute.ca
}

\begin{abstract}
Quasinormal frequencies of electromagnetic and gravitational perturbations in asymptotically AdS spacetime can be identified with poles of the corresponding real-time Green's functions in a holographically dual finite temperature field theory. The quasinormal modes are defined for gauge-invariant quantities which obey incoming-wave boundary condition at the horizon and Dirichlet condition at the boundary. As an application, we explicitly find poles of retarded correlation functions of $R$-symmetry currents and the energymomentum tensor in strongly coupled finite temperature $\mathcal{N}=4$ supersymmetric $S U\left(N_{c}\right)$ Yang-Mills theory in the limit of large $N_{c}$.
\end{abstract}

KEYWORDS: AdS/CFT correspondence, thermal field theory. 


\section{Contents}

1. Introduction 1

2. Field theory correlators 3

2.1 Conserved currents

2.2 Stress-energy tensor 6

3. Quasinormal spectrum and holographic duality 8

3.1 Thermal correlation functions 8

3.2 Black brane fluctuations 10

3.3 Gauge-invariant variables 11

1. Thermal correlators in strongly coupled $\mathcal{N}=4 \mathrm{SYM}$

$4.1 \quad R$-current correlators

4.2 Stress-energy tensor correlators 16

4.2.1 Scalar channel 16

4.2 .2 Shear channel 18

4.2 .3 Sound channel 19

5. Discussion 22

A. Equations of motion and boundary action for the sound channel 24

B. Frobenius solution 25

\section{Introduction}

While the statistical origin of black hole entropy remains a subject of active research, one may wonder if the celebrated analogy [1] between the laws of black hole mechanics and the laws of thermodynamics can be generalized to non-equilibrium processes. Holographic AdS/CFT correspondence ( [2-4], see [5] for a review) provides a suitable arena for such a generalization. AdS/CFT conjecture asserts that string theories on certain asymptotically anti de Sitter spacetimes are dual to quantum field theories in lower dimension. Since the low-energy limit of string theory is described by the appropriate supergravity, problems in general relativity can be mapped to problems in the dual field theory. According to the duality, asymptotically AdS background spacetimes with event horizons are interpreted as thermal states in dual field theories. Correspondingly, small perturbations of a black hole or a black brane background 
are interpreted as small deviations from thermodynamic equilibrium in a dual theory. This particular entry in the holographic dictionary can be made precise by considering quasinormal spectra of asymptotically AdS spacetimes.

Quasinormal modes (see reviews [6] and references therein) are solutions to linearized equations obeyed by classical fluctuations of a gravitational background subject to specific boundary conditions. The choice of the boundary condition at the (future) horizon is dictated by the fact that classically horizons do not emit radiation. Thus out of two local solutions near the horizon typically representing waves incoming to the horizon and outgoing from it, one chooses the incoming waves only. This choice of the boundary condition has profound consequences, making the boundary value problem non-Hermitian, and the associated eigenfrequencies complex. This, however, is exactly what one expects in a holographically dual theory, where small deviations from thermal equilibrium are described by dispersion relations which correspond to non-zero damping [7]. Mathematically, these dispersion relations appear as singularities of the retarded ${ }^{1}$ Green's functions in the complex frequency plane. The connection between quasinormal spectrum of AdS black holes and singularities of thermal correlators in dual quantum field theories was first noted and explored for $2+1$ dimensional BTZ black holes in [8]. It was pointed out later [9] that, even for higher-dimensional systems, imposing Dirichlet boundary conditions for scalar perturbations at asymptotic infinity ensures that quasinormal frequencies coincide with the singularities of the retarded Green's function in a holographically dual theory.

Quasinormal modes for electromagnetic and gravitational perturbations are physically more interesting than those for scalars because the corresponding fluctuations couple to conserved symmetry currents in the dual quantum field theory. However, the relation of these quasinormal modes to correlation functions of the dual theory is not immediate: for example, choosing Dirichlet boundary conditions for gauge-dependent quantities such as metric perturbations would be rather unnatural. Thus, we address the following question in this paper: considering computation of a quasinormal spectrum as a purely general relativity problem (independent of the holographic duality), what variables and boundary conditions should one use in order to ensure that the resulting spectrum coincides with the poles of the correlators in the dual quantum field theory? (For related discussions, see [10-12].)

For vector and gravitational fluctuations, a convenient approach similar to the one used in cosmology [13] is to work with gauge-invariant combinations of the fluctuations. As an example, consider gravitational fluctuations $h_{\mu \nu}$ of five-dimensional AdS-Schwarzschild background with translationally invariant horizon. According to the gauge theory/gravity duality the fluctuation couples to the stress-energy tensor on the boundary [5], and thus we expect the quasinormal spectrum of $h_{\mu \nu}$ to be related to the poles of the retarded two-point correlation function $G_{\mu \nu, \alpha \beta}$ of the stress-energy tensor. As we discuss in Section 2 , the two-point function of the stress-energy tensor in the dual theory is a sum of three independent components

$$
G_{\mu \nu, \alpha \beta}(k)=S_{\mu \nu, \alpha \beta} G_{1}\left(k_{0}, \boldsymbol{k}^{2}\right)+Q_{\mu \nu, \alpha \beta} G_{2}\left(k_{0}, \boldsymbol{k}^{2}\right)+L_{\mu \nu, \alpha \beta} G_{3}\left(k_{0}, \boldsymbol{k}^{2}\right),
$$

\footnotetext{
${ }^{1}$ Choosing outgoing waves at the horizon, one obtains advanced Green's functions in the dual theory.
} 
where $k$ is the four-momentum, and $S_{\mu \nu, \alpha \beta}, Q_{\mu \nu, \alpha \beta}, L_{\mu \nu, \alpha \beta}$ are the appropriate projectors which provide three independent Lorentz index structures. The correlation function is therefore completely determined by three scalar functions $G_{1}, G_{2}, G_{3}$. On the gravity side, one can form three gauge-invariant combinations of the components of $h_{\mu \nu}$ in such a way that each of them corresponds to one of the functions $G_{1}, G_{2}, G_{3}$. Each of these gauge-invariant variables satisfies a second-order ordinary differential equation whose connection matrix essentially determines $G_{1}, G_{2}, G_{3}$. Dirichlet boundary condition imposed on each of the gauge-invariant variables ensures that their quasinormal spectrum corresponds to the poles of $G_{1}, G_{2}, G_{3}$.

The paper is organized as follows. In Section 2 we discuss general Lorentz index structure of thermal correlators of conserved currents and stress-energy tensor in relativistic quantum field theories. In Section 3 we propose a way to identify quasinormal frequencies of asymptotically AdS spacetimes with poles of the corresponding retarded Green's functions in the holographically dual finite temperature field theory. In Section 4 we provide a detailed account of scalar, electromagnetic and gravitational quasinormal spectra for the five dimensional AdS-Schwarzschild background with translationally invariant horizon. Using the approach of gauge-invariant variables, we explicitly show that one can define quasinormal modes whose frequencies coincide with singularities of retarded Green's functions in the dual theory, when the latter are computed using the standard AdS/CFT prescription. In the low-energy limit we reproduce earlier results [14-16] on hydrodynamic properties of $3+1$ dimensional $\mathcal{N}=4$ supersymmetric $S U\left(N_{c}\right)$ Yang-Mills theory. In the more general case, we numerically compute the positions (in the complex frequency plane) of the singularities of retarded correlation functions of global $R$-symmetry currents and energy-momentum tensor in strongly coupled $\mathcal{N}=4$ supersymmetric Yang-Mills theory in the limit of large $N_{c}$. Some technical details appear in two appendices.

\section{Field theory correlators}

We start by discussing Lorentz index structure of retarded Green's functions of conserved currents and energy-momentum tensor in relativistic quantum field theories in infinite, flat $D$-dimensional Minkowski space. Translation and rotation invariance are assumed to be unbroken symmetries of the theory. We shall be interested in retarded Green's functions of conserved symmetry currents,

$$
C_{\mu \nu}(x-y)=-i \theta\left(x^{0}-y^{0}\right)\left\langle\left[J_{\mu}(x), J_{\nu}(y)\right]\right\rangle
$$

as well as of stress-energy tensor,

$$
G_{\mu \nu, \alpha \beta}(x-y)=-i \theta\left(x^{0}-y^{0}\right)\left\langle\left[T_{\mu \nu}(x), T_{\alpha \beta}(y)\right]\right\rangle .
$$

The expectation value is taken in a translation-invariant state, so that the expressions can be Fourier transformed:

$$
C_{\mu \nu}(x-y)=\int \frac{d^{D} k}{(2 \pi)^{D}} e^{i k(x-y)} C_{\mu \nu}(k)
$$


and similarly for $G_{\mu \nu, \alpha \beta}(x-y)$. Here $k=\left(k_{0}, \boldsymbol{k}\right)$ is a $D$-dimensional momentum vector, $k x=k_{0} x^{0}+\boldsymbol{k} \boldsymbol{x}$, and the metric $\eta_{\mu \nu}$ is taken to be mostly plus. Expectation values of all global conserved charges are assumed to vanish in the equilibrium state, in other words we consider systems without chemical potentials. Then CPT invariance of the equilibrium state implies that

$$
\begin{aligned}
& C_{\mu \nu}(k)=C_{\nu \mu}(k), \\
& G_{\mu \nu, \alpha \beta}(k)=G_{\alpha \beta, \mu \nu}(k) .
\end{aligned}
$$

In addition, correlation functions of stress-energy tensor satisfy

$$
G_{\mu \nu, \alpha \beta}(k)=G_{\nu \mu, \alpha \beta}(k)=G_{\mu \nu, \beta \alpha}(k)
$$

because of the symmetry of $T_{\mu \nu}(x)$. Conservation of $J_{\mu}(x)$ and $T_{\mu \nu}(x)$ implies that the correlation functions may be defined so that they satisfy the following Ward identities ${ }^{2}$

$$
\begin{aligned}
& k^{\mu} C_{\mu \nu}(k)=0, \\
& k^{\mu} G_{\mu \nu, \alpha \beta}(k)=0 .
\end{aligned}
$$

If in addition the theory possesses scale invariance, then the correlation functions of stressenergy tensors satisfy an extra Ward identity

$$
\eta^{\mu \nu} G_{\mu \nu, \alpha \beta}(k)=0
$$

Hermiticity of $J_{\mu}$ and $T_{\mu \nu}$ combined with rotation invariance implies

$$
\begin{aligned}
& C_{\mu \nu}\left(k_{0}, \boldsymbol{k}\right)=C_{\mu \nu}\left(-k_{0}, \boldsymbol{k}\right)^{*}, \\
& G_{\mu \nu, \alpha \beta}\left(k_{0}, \boldsymbol{k}\right)=G_{\mu \nu, \alpha \beta}\left(-k_{0}, \boldsymbol{k}\right)^{*} .
\end{aligned}
$$

\subsection{Conserved currents}

In vacuum, the Ward identity (2.7) implies that the current-current correlation function $C_{\mu \nu}(k)$ is proportional to the projector onto conserved vectors,

$$
P_{\mu \nu}=\eta_{\mu \nu}-\frac{k_{\mu} k_{\nu}}{k^{2}}
$$

where $k^{2}=-k_{0}^{2}+\boldsymbol{k}^{2}$. All components of $C_{\mu \nu}(k)$ are thus determined by a single scalar function,

$$
C_{\mu \nu}(k)=P_{\mu \nu} \Pi\left(k^{2}\right) .
$$

\footnotetext{
${ }^{2}$ One may choose to define the correlation functions in such a way that local (in position space) counterterms appear on the right-hand side of the Ward identities. The correlation functions defined in this way will differ from $C_{\mu \nu}(k)$ and $G_{\mu \nu, \alpha \beta}(k)$ by analytic functions of $k_{0}$ and $\boldsymbol{k}$.
} 
If the expectation value is taken in a state that has only rotation symmetry (such as thermal equilibrium state in canonical ensemble), it is convenient to split the projector $P_{\mu \nu}$ into transverse and longitudinal parts,

$$
P_{\mu \nu}=P_{\mu \nu}^{T}+P_{\mu \nu}^{L}
$$

where $P_{\mu \nu}^{T}$ and $P_{\mu \nu}^{L}$ are mutually orthogonal $\left(P_{\alpha \mu}^{T} \eta^{\mu \nu} P_{\nu \beta}^{L}=0\right)$ projectors defined as

$$
\begin{aligned}
& P_{00}^{T}=0, \quad P_{0 i}^{T}=0, \quad P_{i j}^{T}=\delta_{i j}-\frac{k_{i} k_{j}}{\boldsymbol{k}^{2}}, \\
& P_{\mu \nu}^{L}=P_{\mu \nu}-P_{\mu \nu}^{T} .
\end{aligned}
$$

They satisfy $k^{\mu} P_{\mu \nu}^{T}=k^{\mu} P_{\mu \nu}^{L}=0$, and therefore any expression constructed out of $P_{\mu \lambda}^{T}, P_{\mu \lambda}^{L}$ will automatically satisfy current-conservation constraint. Therefore in the rotation-invariant case the current-current correlation function is determined by two independent scalar functions,

$$
C_{\mu \nu}(k)=P_{\mu \nu}^{T} \Pi^{T}\left(k_{0}, \boldsymbol{k}^{2}\right)+P_{\mu \nu}^{L} \Pi^{L}\left(k_{0}, \boldsymbol{k}^{2}\right)
$$

When $\Pi^{T}=\Pi^{L}=\Pi$, this expression reduces to the Lorentz-invariant form (2.13). Also, it is not difficult to see that due to rotation invariance the $\boldsymbol{k} \rightarrow 0$ limits (with $k_{0}$ fixed) of transverse and longitudinal self-energies coincide,

$$
\lim _{\boldsymbol{k} \rightarrow 0} \Pi^{T}\left(k_{0}, \boldsymbol{k}^{2}\right)=\lim _{\boldsymbol{k} \rightarrow 0} \Pi^{L}\left(k_{0}, \boldsymbol{k}^{2}\right) .
$$

As an example, consider a four-dimensional field theory at non-zero temperature. Without loss of generality one can take the spatial momentum oriented along the $x^{3}$ direction, so that $k_{\mu}=(-\omega, 0,0, q)$, with $k^{2}=-\omega^{2}+q^{2}$. Then the components of the current-current correlation function are

$$
C_{x^{1} x^{1}}(k)=C_{x^{2} x^{2}}(k)=\Pi^{T}(\omega, q)
$$

as well as

$$
C_{t t}(k)=\frac{q^{2}}{\omega^{2}-q^{2}} \Pi^{L}(\omega, q), \quad C_{t x^{3}}(k)=\frac{-\omega q}{\omega^{2}-q^{2}} \Pi^{L}(\omega, q), \quad C_{x^{3} x^{3}}(k)=\frac{\omega^{2}}{\omega^{2}-q^{2}} \Pi^{L}(\omega, q) .
$$

For a system in stable thermodynamic equilibrium at temperature $T$, the low-energy $(\omega \ll T$, $q \ll T$ ) behavior of $\Pi^{T}$ and $\Pi^{L}$ is universal and is described by effective hydrodynamic theory (see for example [17]). In the approximation of linearized hydrodynamics, $\Pi^{T}(\omega, q)$ is nonsingular as a function of $\omega$ because it does not couple to charge density fluctuations. On the other hand, correlators which involve conserved charge density must exhibit a hydrodynamic singularity whose dispersion relation satisfies $\omega(q) \rightarrow 0$ as $q \rightarrow 0$. The longitudinal self-energy $\Pi^{L}(\omega, q)$ has a simple pole at $\omega=-i D_{\mathrm{Q}} q^{2}$, where $D_{\mathrm{Q}}$ is the diffusion constant of charge $Q$ associated with current $J_{\mu}(x)$. 


\subsection{Stress-energy tensor}

In vacuum, the two-point correlation function of stress-energy tensor may be written as a sum of five terms allowed by the symmetries (2.5), (2.6), which are proportional to $\eta_{\mu \nu} \eta_{\alpha \beta}$, $\left(\eta_{\mu \alpha} \eta_{\nu \beta}+\eta_{\mu \beta} \eta_{\nu \alpha}\right),\left(\eta_{\mu \nu} k_{\alpha} k_{\beta}+k_{\mu} k_{\nu} \eta_{\alpha \beta}\right),\left(\eta_{\mu \alpha} k_{\nu} k_{\beta}+\eta_{\nu \alpha} k_{\mu} k_{\beta}+\eta_{\mu \beta} k_{\nu} k_{\alpha}+\eta_{\nu \beta} k_{\mu} k_{\alpha}\right)$, and $k_{\mu} k_{\nu} k_{\alpha} k_{\beta}$. Only two linear combinations of these terms are consistent with the Ward identity (2.8); they can be taken to be $P_{\mu \nu} P_{\alpha \beta}$ and $P_{\mu \alpha} P_{\nu \beta}+P_{\mu \beta} P_{\nu \alpha}$. A convenient way to write $G_{\mu \nu, \alpha \beta}(k)$ is

$$
G_{\mu \nu, \alpha \beta}(k)=P_{\mu \nu} P_{\alpha \beta} G_{B}\left(k^{2}\right)+H_{\mu \nu, \alpha \beta} G_{S}\left(k^{2}\right)
$$

where

$$
H_{\mu \nu, \alpha \beta}=\frac{1}{2}\left(P_{\mu \alpha} P_{\nu \beta}+P_{\mu \beta} P_{\nu \alpha}\right)-\frac{1}{D-1} P_{\mu \nu} P_{\alpha \beta}
$$

is a projector onto conserved traceless symmetric tensors, which is constructed to satisfy $\eta^{\mu \nu} H_{\mu \nu, \alpha \beta}=0$. As a result, a scale-invariant theory must have $G_{B}\left(k^{2}\right)=0$, and the correlation function takes a simple form

$$
G_{\mu \nu, \alpha \beta}(k)=H_{\mu \nu, \alpha \beta} G_{S}\left(k^{2}\right) .
$$

If the expectation value is taken in a state that has only rotation symmetry (such as thermal equilibrium state in the canonical ensemble), it is convenient to split $H_{\mu \nu, \alpha \beta}$ into mutually orthogonal projectors constructed out of $P_{\mu \nu}^{T}, P_{\mu \nu}^{L}$. One relevant combination is

$$
S_{\mu \nu, \alpha \beta}=\frac{1}{2}\left(P_{\mu \alpha}^{T} P_{\nu \beta}^{L}+P_{\mu \alpha}^{L} P_{\nu \beta}^{T}+P_{\mu \beta}^{T} P_{\nu \alpha}^{L}+P_{\mu \beta}^{L} P_{\nu \alpha}^{T}\right)
$$

It satisfies $k^{\mu} S_{\mu \nu, \alpha \beta}=0$, and also $\eta^{\mu \nu} S_{\mu \nu, \alpha \beta}=0$ because of the orthogonality of $P^{T}$ and $P^{L}$. It is not difficult to find another independent combination with the same properties,

$$
Q_{\mu \nu, \alpha \beta}=\frac{1}{D-1}\left((D-2) P_{\mu \nu}^{L} P_{\alpha \beta}^{L}+\frac{1}{D-2} P_{\mu \nu}^{T} P_{\alpha \beta}^{T}-\left(P_{\mu \nu}^{T} P_{\alpha \beta}^{L}+P_{\mu \nu}^{L} P_{\alpha \beta}^{T}\right)\right) .
$$

The projectors $S_{\mu \nu, \alpha \beta}$ and $Q_{\mu \nu, \alpha \beta}$ square to themselves, and are orthogonal to each other $\left(S_{\mu \nu, \alpha \beta} \eta^{\alpha \lambda} \eta^{\beta \rho} Q_{\lambda \rho, \sigma \tau}=0\right)$. Therefore the projector $H_{\mu \nu, \alpha \beta}$ can be split as

$$
H_{\mu \nu, \alpha \beta}=S_{\mu \nu, \alpha \beta}+Q_{\mu \nu, \alpha \beta}+L_{\mu \nu, \alpha \beta}
$$

where $L_{\mu \nu, \alpha \beta} \equiv H_{\mu \nu, \alpha \beta}-S_{\mu \nu, \alpha \beta}-Q_{\mu \nu, \alpha \beta}$ is orthogonal to both $S_{\mu \nu, \alpha \beta}$ and $Q_{\mu \nu, \alpha \beta}$. Thus the correlation function of energy-momentum tensor in a scale-invariant theory can be written as a sum over three independent index structures,

$$
G_{\mu \nu, \alpha \beta}(k)=S_{\mu \nu, \alpha \beta} G_{1}\left(k_{0}, \boldsymbol{k}^{2}\right)+Q_{\mu \nu, \alpha \beta} G_{2}\left(k_{0}, \boldsymbol{k}^{2}\right)+L_{\mu \nu, \alpha \beta} G_{3}\left(k_{0}, \boldsymbol{k}^{2}\right) .
$$

It is not difficult to show that rotation invariance implies that $\boldsymbol{k} \rightarrow 0$ limits (with $k_{0}$ fixed) of the three independent scalar functions must coincide,

$$
\lim _{\boldsymbol{k} \rightarrow 0} G_{1}\left(k_{0}, \boldsymbol{k}^{2}\right)=\lim _{\boldsymbol{k} \rightarrow 0} G_{2}\left(k_{0}, \boldsymbol{k}^{2}\right)=\lim _{\boldsymbol{k} \rightarrow 0} G_{3}\left(k_{0}, \boldsymbol{k}^{2}\right) .
$$


In a scale non-invariant theory, two extra scalar functions are needed to specify $G_{\mu \nu, \alpha \beta}(k)$. They multiply two independent linear combinations of $P_{\mu \nu}^{T} P_{\alpha \beta}^{T}, P_{\mu \nu}^{L} P_{\alpha \beta}^{L}$, and $\left(P_{\mu \nu}^{T} P_{\alpha \beta}^{L}+\right.$ $\left.P_{\mu \nu}^{L} P_{\alpha \beta}^{T}\right)$; one possible choice is

$$
\begin{aligned}
G_{\mu \nu, \alpha \beta}(k) & =\left(P_{\mu \nu}^{T} P_{\alpha \beta}^{T}+\frac{1}{2}\left(P_{\mu \nu}^{T} P_{\alpha \beta}^{L}+P_{\mu \nu}^{L} P_{\alpha \beta}^{T}\right)\right) C_{T}\left(k_{0}, \boldsymbol{k}^{2}\right) \\
& +\left(P_{\mu \nu}^{L} P_{\alpha \beta}^{L}+\frac{1}{2}\left(P_{\mu \nu}^{T} P_{\alpha \beta}^{L}+P_{\mu \nu}^{L} P_{\alpha \beta}^{T}\right)\right) C_{L}\left(k_{0}, \boldsymbol{k}^{2}\right) \\
& +S_{\mu \nu, \alpha \beta} G_{1}\left(k_{0}, \boldsymbol{k}^{2}\right)+Q_{\mu \nu, \alpha \beta} G_{2}\left(k_{0}, \boldsymbol{k}^{2}\right)+L_{\mu \nu, \alpha \beta} G_{3}\left(k_{0}, \boldsymbol{k}^{2}\right) .
\end{aligned}
$$

When $C_{T}=C_{L}=G_{B}$ and $G_{1}=G_{2}=G_{3}=G_{S}$, this expression reduces to the Lorentz-invariant form (2.21).

As an example, consider a four-dimensional field theory at non-zero temperature. Choosing momentum to be $k_{\mu}=(-\omega, 0,0, q)$ as above, one finds the following components of the correlation function. The correlations of transverse momentum density are determined by $G_{1}(\omega, q)$

$$
\begin{aligned}
& G_{t x^{1}, t x^{1}}(k)=\frac{1}{2} \frac{q^{2}}{\omega^{2}-q^{2}} G_{1}(\omega, q), \\
& G_{t x^{1}, x^{1} x^{3}}(k)=-\frac{1}{2} \frac{\omega q}{\omega^{2}-q^{2}} G_{1}(\omega, q), \\
& G_{x^{1} x^{3}, x^{1} x^{3}}(k)=\frac{1}{2} \frac{\omega^{2}}{\omega^{2}-q^{2}} G_{1}(\omega, q) .
\end{aligned}
$$

The correlations of longitudinal momentum density, energy density, and diagonal stress are determined by $G_{2}(\omega, q), C_{L}(\omega, q), C_{T}(\omega, q)$. For example,

$$
\begin{aligned}
& G_{t t, t t}(k)=\frac{1}{3} \frac{q^{4}}{\left(\omega^{2}-q^{2}\right)^{2}}\left[2 G_{2}(\omega, q)+3 C_{L}(\omega, q)\right], \\
& G_{t t, t x^{3}}(k)=-\frac{1}{3} \frac{\omega q^{3}}{\left(\omega^{2}-q^{2}\right)^{2}}\left[2 G_{2}(\omega, q)+3 C_{L}(\omega, q)\right], \\
& G_{t t, x^{1} x^{1}}=\frac{1}{6} \frac{q^{2}}{\left(q^{2}-\omega^{2}\right)}\left[2 G_{2}(\omega, q)-3 C_{L}(\omega, q)-3 C_{T}(\omega, q)\right] .
\end{aligned}
$$

The correlations of transverse stress are determined by $G_{3}(\omega, q)$,

$$
G_{x^{1} x^{2}, x^{1} x^{2}}(k)=\frac{1}{2} G_{3}(\omega, q) .
$$

For a system in stable thermodynamic equilibrium at temperature $T$, the low-energy $(\omega \ll T$, $q \ll T)$ behavior of $G_{\mu \nu, \alpha \beta}$ is universal and is described by effective hydrodynamic theory (see for example [17]). In the approximation of linearized hydrodynamics, $G_{3}(\omega, q)$ is nonsingular as a function of $\omega$ because it does not couple to energy density or momentum density fluctuations. On the other hand, correlation functions which involve conserved densities exhibit hydrodynamic singularities whose dispersion relations satisfy $\omega(q) \rightarrow 0$ as $q \rightarrow 0$. Function 
$G_{1}(\omega, q)$ has a simple pole at $\omega=-i \gamma_{\eta} q^{2}$, where $\gamma_{\eta}$ is the damping constant of the shear mode, proportional to shear viscosity. In a conformal theory (when $C_{L}=C_{T}=0$ ), function $G_{2}(\omega, q)$ has simple poles at $\omega= \pm v_{s} q-i \Gamma_{s} q^{2}$, where $v_{s}$ is the speed of sound, and $\Gamma_{s}$ is the damping constant of the sound mode, also proportional to shear viscosity.

\section{Quasinormal spectrum and holographic duality}

\subsection{Thermal correlation functions}

We will be interested in small fluctuations of a black $p$-brane,

$$
d s^{2}=a(r)\left(-f(r) d t^{2}+\sum_{i=1}^{p}\left(d x^{i}\right)^{2}\right)+b(r) d r^{2} .
$$

Metrics of this form arise as a result of dimensional reduction of higher dimensional supergravity backgrounds. In addition, these backgrounds have non-zero values of various "matter" fields which we collectively denote by $\phi^{(0)}$ suppressing all indices. Holographically dual theory is defined on the boundary $(r \rightarrow \infty)$ of (3.1), which is a flat $p+1$ dimensional Minkowski space.

Translation invariance on the boundary implies that all fluctuating fields can be taken to be proportional to $e^{-i \omega t+i \boldsymbol{q} \boldsymbol{x}}$; thus linearized fluctuations $\delta g_{\mu \nu}, \delta \phi$ of the background will obey a system of second-order linear ordinary differential equations. Generically, the system will be redundant, reflecting the gauge freedom (such as linearized diffeomorphisms) enjoyed by the fluctuation fields. Instead of fixing a particular gauge, we consider gauge-invariant combinations of the fluctuation fields. Let $Z_{k}$ be these gauge-invariant variables which are constructed as linear combinations of fluctuating fields and their derivatives, excluding $r$ derivatives. Variables $Z_{k}$ will obey a system of coupled second order linear ordinary differential equations (ODEs) which can in principle be diagonalized. Let $Z(r)$ be such a gauge-invariant variable satisfying a second-order ODE. A local solution of the ODE near the horizon will generally be a superposition of incoming and outgoing waves. Since classically the horizon does not radiate, we choose the incoming wave boundary condition there.

The solution obeying the incoming wave boundary condition at the horizon can be written in the basis of two local solutions at the boundary ${ }^{3}$ as

$$
Z(r)=\mathcal{A} \varphi_{1}(r)+\mathcal{B} \varphi_{2}(r)
$$

where $\mathcal{A}, \mathcal{B}$ are the connection coefficients of the corresponding ODE. Coefficients $\mathcal{A}, \mathcal{B}$ typically depend on the parameters (such as frequency and momentum) which enter the differential equation for $Z(r)$. Near the boundary, the solution (3.2) becomes

$$
Z(r)=\mathcal{A} r^{-\Delta_{-}}(1+\cdots)+\mathcal{B} r^{-\Delta_{+}}(1+\cdots)
$$

\footnotetext{
${ }^{3}$ By local solutions we mean the solutions obtained as power series around the corresponding singular point $(r=\infty$ in this case) of the differential equation. See for example [18] for a general discussion.
} 
where $\Delta_{+}, \Delta_{-}$are exponents of the ODE at $r=\infty$, and ellipses denote higher powers of $r$. We consider the situation when the exponents are not equal, $\Delta_{+}>\Delta_{-}$, and $\Delta_{+}$can be taken positive. ${ }^{4}$

The action of the system expanded to quadratic order in fluctuations $\delta g_{\mu \nu}, \delta \phi$ can be rewritten in terms of the gauge-invariant variables. On shell, the part of the action quadratic in fluctuations will reduce to the boundary term

$$
S^{(2)} \sim \lim _{r \rightarrow \infty} \int d \omega d^{p} q F(\omega, \boldsymbol{q}) Z^{\prime}(r) Z(r)+\text { contact terms },
$$

where $F(\omega, \boldsymbol{q})$ depends on the details of the action, and "contact terms" do not contain $Z^{\prime}(r)$.

In holographic AdS/CFT duality, fluctuation $\delta \phi$ couples to a particular operator $\mathcal{O}$ of the dual theory at the boundary. Applying the Lorentzian AdS/CFT prescription $[9,20]$ to the action (3.4) to compute the retarded correlator, and remembering that $Z(r)$ is a functional of $\delta \phi$, we find

$$
\langle\mathcal{O O}\rangle_{R} \sim \frac{\mathcal{B}}{\mathcal{A}}+\text { contact terms }
$$

The poles of the retarded correlator correspond to zeros of the connection coefficient $\mathcal{A}$. On the other hand, setting $\mathcal{A}=0$ in Eq. (3.2) corresponds to a particular choice of boundary conditions for the fluctuation $Z(r)$. From the general relativity point of view, this choice determines the quasinormal spectrum of $Z(r)$. In other words, equation $\mathcal{A}=0$ defines quasinormal spectrum for gauge-invariant perturbations which has the interpretation of the poles of retarded correlators in a holographically dual theory. ${ }^{5}$ This argument is similar to the one given in $[9,22]$ in the case of a scalar fluctuation, the difference being the use of gauge-invariant variables in the present discussion.

\footnotetext{
${ }^{4}$ In AdS/CFT correspondence, $\Delta_{+}$is equal to the conformal dimension of the operator that couples to bulk fields contained in $Z$. See also [19] for exceptional cases.

${ }^{5}$ One should add at once that in addition to zeros of $\mathcal{A}$, singularities of the correlator (3.5) may also come from singularities of $\mathcal{B}$. However, singularities of $\mathcal{B}$ are completely determined by the singularities of the local Frobenius solution $\varphi_{1}$ considered as a function of parameter(s) of the differential equation. Indeed, a general theorem [21] guarantees smoothness of a solution of a differential equation with respect to a parameter, if the equation and the boundary conditions depend smoothly on the parameter. Thus the solution $Z$ is smooth, and singularities of $\mathcal{B}$ are destined to cancel the singularities of the coefficients of the series expansion in the local Frobenius solution $\varphi_{1}$ with respect to a parameter. Therefore, singularities of $\mathcal{B}$ are essentially determined by the recursion relations for the coefficients of the series expansion which defines $\varphi_{1}$. Let us illustrate this point using hypergeometric equation as an example. On the interval $z \in[0,1]$, the solution ${ }_{2} F_{1}(a, b ; c ; z)$ defined by its recursion relations at $z=0$ is related to two local solutions at $z=1$ by

$$
\begin{aligned}
{ }_{2} F_{1}(a, b ; c ; z) & =\mathcal{A}_{2} F_{1}(a, b ; a+b-c+1 ; 1-z) \\
& +\mathcal{B}(1-z)^{c-a-b}{ }_{2} F_{1}(c-a, c-b ; c-a-b+1 ; 1-z),
\end{aligned}
$$

where the connection matrix coefficients are given by

$$
\mathcal{A}=\frac{\Gamma(c) \Gamma(c-a-b)}{\Gamma(c-a) \Gamma(c-b)}, \quad \mathcal{B}=\frac{\Gamma(c) \Gamma(a+b-c)}{\Gamma(a) \Gamma(b)} .
$$

To be in agreement with the scenario set by Eq. (3.2), let us further assume that $c-a-b>0$. The poles of the correlator (3.5) come from the poles of $\Gamma(c-a)$ and $\Gamma(c-b)$ (also corresponding to the Dirichlet condition
} 


\subsection{Black brane fluctuations}

According to the dictionary of the AdS/CFT correspondence [5], global symmetry currents of the dual field theory have as their sources boundary values of the gauge field $A_{\mu}$ on the higherdimensional background (3.1). Similarly, stress-energy tensor of the dual theory is sourced by gravitational fluctuations $h_{\mu \nu}$ of the black brane. We shall be interested in quasinormal spectra of these fluctuations. We take the fluctuations to be of the form $A_{\mu}(r) e^{-i \omega t+i q z}$, $h_{\mu \nu}(r) e^{-i \omega t+i q z}$ where $z=x^{p}$. The fluctuations can be classified according to their transformation properties under the "remaining" world-volume symmetry group $O(p-1)$ acting on $x^{1}, . ., x^{p-1}$.

Let us restrict ourselves to the simplest case when the considered fluctuations do not couple to fluctuations of other background fields. Components $A_{t}, A_{z}$ do not transform under $O(p-1)$, while components $A_{\alpha}, \alpha=x^{1}, . ., x^{p-1}$ transform as vectors. One can therefore distinguish two symmetry channels for electromagnetic fluctuations

$$
\begin{aligned}
& \text { Spin } 0 \text { (diffusive channel): } \quad A_{t}, A_{z}, A_{r} \\
& \text { Spin } 1 \text { (transverse channel): } A_{\alpha} \text {. }
\end{aligned}
$$

A similar classification can be adopted for metric fluctuations. Components $h_{t t}, h_{t z}, h_{z z}, h_{r r}$, $h_{t r}$, and $h_{z r}$ do not transform under $O(p-1)$, components $h_{t \alpha}, h_{z \alpha}$, and $h_{r \alpha}$ transform as vectors, while $h_{\alpha \beta}$ transform as rank-2 tensors. The tensor representation is reducible, for a symmetric $h_{\alpha \beta}$ can be decomposed into the trace part $\delta_{\alpha \beta} h /(p-1)$, where $h=\sum_{\alpha} h_{\alpha \alpha}$ (a singlet) and the symmetric traceless part $h_{\alpha \beta}-\delta_{\alpha \beta} h /(p-1)$. Thus we have three symmetry channels for gravitational fluctuations: ${ }^{6}$

$$
\begin{array}{ll}
\text { Spin } 0 \text { (sound channel): } & h_{t t}, h_{t z}, h_{z z}, h, h_{r r}, h_{t r}, h_{z r} \\
\text { Spin } 1 \text { (shear channel): } & h_{t \alpha}, h_{z \alpha}, h_{r \alpha} \\
\text { Spin } 2 \text { (scalar channel): } & h_{\alpha \beta}-\delta_{\alpha \beta} h /(p-1) .
\end{array}
$$

The $O(p-1)$ symmetry guarantees that equations for fluctuations belonging to different symmetry channels decouple. Classification presented here mirrors the classification of the correlators in Section 2: diffusive and transverse channels of the $U(1)$ fluctuation correspond

$\mathcal{A}=0)$, and from the poles of $\Gamma(a+b-c)$. The latter are determined by the local solution near $z=1$,

$$
\begin{aligned}
{ }_{2} F_{1}(a, b ; a+b-c+1 ; 1-z) & =1+\frac{a b}{1+a+b-c}(1-z) \\
& +\frac{a b(a+1)(b+1)}{2(1+a+b-c)(2+a+b-c)}(1-z)^{2}+\cdots,
\end{aligned}
$$

the coefficients of which have poles at $a+b-c=-n$, where $n$ is a positive integer. These are precisely the poles of the correlator 3.5) coming from $\mathcal{B}$. By setting $a+b-c=-n+\epsilon$ and taking the limit $\epsilon \rightarrow 0$, one can show that the right hand side of Eq. (3.6) is in fact a smooth function of the parameters $a, b, c$.

${ }^{6}$ The name "scalar" for spin-2 fluctuations reflects the fact that the corresponding wave equation coincides with that of the minimally coupled massless scalar [23]. The names "shear" and "sound" (as well as "diffusive" above) reflect physical interpretation of the lowest quasinormal frequency (for a given symmetry channel) in the dual field theory. This will be seen explicitly in the next section. 
to functions $\Pi^{L}$ and $\Pi^{T}$ of the current-current correlator. When the dual theory possesses conformal invariance, shear, sound and scalar channels of the gravitational fluctuation are related to functions $G_{1}, G_{2}, G_{3}$ of the stress-energy tensor correlator.

\subsection{Gauge-invariant variables}

We now define gauge-invariant variables corresponding to classes (3.7), (3.8). Combinations of the gauge field components invariant under the transformation $A_{\mu} \rightarrow A_{\mu}-\partial_{\mu} \lambda$ include components of the electric field

$$
\begin{aligned}
\text { Diffusive channel: } & E_{z}=q A_{t}+\omega A_{z}, \\
\text { Transverse channel: } & E_{\alpha}=\omega A_{\alpha} .
\end{aligned}
$$

Perturbations $h_{\mu \nu}$ transform under infinitesimal diffeomorphisms as $h_{\mu \nu} \rightarrow h_{\mu \nu}-\nabla_{\mu} \xi_{\nu}-\nabla_{\nu} \xi_{\mu}$, where $\xi_{\mu}=\xi_{\mu}(r) e^{-i \omega t+i q z}$ are gauge functions, and covariant derivatives are taken with respect to the background metric (3.1). One may define the following gauge-invariant combinations linear in perturbations:

Shear channel: $Z_{1}=q H_{t x^{1}}+\omega H_{z x^{1}}$

Sound channel: $Z_{2}=q^{2} f H_{t t}+2 \omega q H_{t z}+\omega^{2} H_{z z}+q^{2} f\left(1+\frac{a f^{\prime}}{a^{\prime} f}-\frac{\omega^{2}}{q^{2} f}\right) H$

Scalar channel: $Z_{3}=H_{x^{1} x^{2}}$,

where $H_{t t}=h_{t t} / a f, H_{t z}=h_{t z} / a, H_{i j}=h_{i j} / a(i, j \neq t), H=h /(p-1) a$. From Einstein equations obeyed by the fluctuations, one obtains three independent second-order ODEs satisfied by $Z_{1}, Z_{2}$, and $Z_{3}$.

Quasinormal modes are defined as solutions to the second-order differential equations satisfied by the gauge-invariant variables $E_{z}, E_{\alpha}, Z_{1}, Z_{2}, Z_{3}$ obeying incoming wave boundary condition at the horizon and Dirichlet condition at the boundary. According to the above discussion, the spectra of complex eigenfrequencies obtained in solving the boundary value problem for fluctuations $E_{z}, E_{\alpha}, Z_{1}, Z_{2}, Z_{3}$ coincide with poles of the functions $\Pi^{L}, \Pi^{T}, G_{1}$, $G_{2}, G_{3}$, respectively.

\section{Thermal correlators in strongly coupled $\mathcal{N}=4 \mathrm{SYM}$}

We now apply the approach outlined in Section 3 to the near-horizon limit of the non-extremal gravitational background of type IIB low energy string theory describing $N_{c}$ parallel black three-branes. The background is given by the metric

$$
d s^{2}=\frac{r^{2}}{R^{2}}\left(-f(r) d t^{2}+d x^{2}+d y^{2}+d z^{2}\right)+\frac{R^{2}}{r^{2} f(r)} d r^{2}+R^{2} d \Omega_{5}^{2},
$$

where $R$ is a constant, which depends on the number of $D 3$ branes, $R \propto N_{c}^{1 / 4}$, and $f(r)=$ $1-r_{0}^{4} / r^{4}$. The parameter of non-extremality $r_{0}$ specifies the location of the horizon, whose 
Hawking temperature is $T=r_{0} / \pi R^{2}$. Upon dimensional reduction on $S^{5}$, gravitational perturbations will effectively propagate on the five-dimensional part of the background (4.1). Introducing new coordinate $u=r_{0}^{2} / r^{2}$, the metric can be written as

$$
d s^{2}=\frac{(\pi T R)^{2}}{u}\left(-f(u) d t^{2}+d x^{2}+d y^{2}+d z^{2}\right)+\frac{R^{2}}{4 u^{2} f(u)} d u^{2},
$$

where $f(u)=1-u^{2}$. In these coordinates, the horizon is located at $u=1$, and the boundary is at $u=0$. In addition, the background is specified by the value of the self-dual five form field

$$
F_{5}=\frac{2(\pi T R)^{4}}{u^{3}}(1+*) d t \wedge d x \wedge d y \wedge d z \wedge d u
$$

with all other fields vanishing. The dual quantum field theory is $\mathcal{N}=4 S U\left(N_{c}\right)$ supersymmetric Yang-Mills theory in $3+1$ dimensional Minkowski space at large $N_{c}$ and large 't Hooft coupling [2]. The field theory is taken in a thermal equilibrium state at a temperature equal to the Hawking temperature of the background. Real-time thermal correlators of the conserved $R$-symmetry currents and stress-energy tensor in this theory were considered in the AdS/CFT approach in $[11,15,16]$. Here we show that reformulating the problem in terms of gauge-invariant variables allows one to compute functions $\Pi^{L}, \Pi^{T}, G_{1}, G_{2}, G_{3}$ directly by solving the second-order ODEs associated with each of them. In particular, quasinormal spectra of the gauge-invariant fluctuations determine poles of these functions in complex frequency plane. ${ }^{7}$

\subsection{R-current correlators}

Correlators of $R$-currents in strongly coupled $\mathcal{N}=4 \mathrm{SYM}$ at zero temperature were computed in $[24,25]$ by using the AdS/CFT correspondence. Similar approach can be taken at non-zero temperature [15]. On the gravity side of AdS/CFT, one considers an effective $U(1)$ field ${ }^{8}$ in the five-dimensional asymptotically AdS part of the background (4.2). This five-dimensional Maxwell field is essentially a graviphoton of the dimensional reduction of (4.1) on $S^{5}$.

According to the discussion in Section 2, thermal current-current correlators are determined by two independent scalar functions, $\Pi^{T}(\omega, q)$ and $\Pi^{L}(\omega, q)$. Correspondingly, we expect the dual five-dimensional Maxwell system to reduce to two independent equations for gauge-invariant variables whose quasinormal frequencies determine the poles of the correlators. One of the quasinormal frequencies should be purely imaginary (at least in the regime $q / T \ll 1$ ), reflecting diffusive relaxation of large-scale charge density fluctuations around thermal equilibrium state in the dual field theory.

Maxwell's equations for the $U(1)$ field are simply $\partial_{A}\left(\sqrt{-g} g^{A C} g^{B D} F_{C D}\right)=0$, where $F_{C D}=$ $\partial_{C} A_{D}-\partial_{D} A_{C}$, capital Latin indices run over $t, x, y, z, u$, and components of the five dimensional metric $g_{A B}$ are given by (4.2). Translation invariance for the $t, x, y, z$ coordinates implies

\footnotetext{
${ }^{7}$ The problem of additional singularities discussed in footnote $\mathrm{\theta}$ does not arise here.

${ }^{8}$ The $R$-charges of the theory $Q^{a}=\int d^{3} x j_{0}^{a}(x)$ generate global $S U(4)$ symmetry group, with $a=1 \ldots 15$. In an equilibrium state without chemical potentials for the $R$-charges, the correlation function of $R$ currents $j_{\mu}^{a}$ has the form $C_{\mu \nu}^{a b}(x)=\delta^{a b} C_{\mu \nu}(x)$. The expressions of this section refer to $C_{\mu \nu}(x)$.
} 
that vector potential can be Fourier transformed,

$$
A_{C}(u, t, \boldsymbol{x})=\int \frac{d^{4} k}{(2 \pi)^{4}} e^{i k_{0} t+i \boldsymbol{k} \boldsymbol{x}} A_{C}(u, k) .
$$

Choosing $k=(-\omega, 0,0, q)$, one can derive the following equations satisfied by transverse and longitudinal electric fields:

$$
\begin{aligned}
& E_{\alpha}^{\prime \prime}+\frac{f^{\prime}}{f} E_{\alpha}^{\prime}+\frac{\mathfrak{w}^{2}-\mathfrak{q}^{2} f}{u f^{2}} E_{\alpha}=0, \quad \alpha=x, y, \\
& E_{z}^{\prime \prime}+\frac{\mathfrak{w}^{2} f^{\prime}}{f\left(\mathfrak{w}^{2}-\mathfrak{q}^{2} f\right)} E_{z}^{\prime}+\frac{\mathfrak{w}^{2}-\mathfrak{q}^{2} f}{u f^{2}} E_{z}=0,
\end{aligned}
$$

where $E_{\alpha} \equiv \mathfrak{w} A_{\alpha}, E_{z} \equiv \mathfrak{q} A_{t}+\mathfrak{w} A_{z}$, dimensionless parameters $\mathfrak{w}$ and $\mathfrak{q}$ are defined as

$$
\mathfrak{w}=\frac{\omega}{2 \pi T}, \quad \mathfrak{q}=\frac{q}{2 \pi T},
$$

and prime denotes the derivative with respect to $u$.

Gauge-gravity duality implies that all information about two-point $R$-current correlation functions in the dual $\mathcal{N}=4 \mathrm{SYM}$ theory (in the large $N_{c}$ and large 't Hooft coupling limit) is contained in the solutions to the differential equations (4.5). For both equations, the singularity at $u=1$ (the horizon) has exponents $\pm i \mathfrak{w} / 2$ corresponding to the outgoing/incoming waves. To compute the retarded correlators, one has to impose the incoming wave boundary condition at the horizon [9] thus choosing $-i \mathfrak{w} / 2$ as the correct exponent. At the boundary $(u=0)$ the exponents for both equations are 0 and 1 , and thus solutions to equations (4.5) which satisfy incoming-wave boundary condition at the horizon behave near $u=0$ as

$$
\begin{aligned}
E_{\alpha}(u) & =\mathcal{A}_{(\alpha)}(\mathfrak{w}, \mathfrak{q})+\cdots+\mathcal{B}_{(\alpha)}(\mathfrak{w}, \mathfrak{q}) u+\cdots \\
E_{z}(u) & =\mathcal{A}_{(z)}(\mathfrak{w}, \mathfrak{q})+\cdots+\mathcal{B}_{(z)}(\mathfrak{w}, \mathfrak{q}) u+\cdots
\end{aligned}
$$

The boundary action of the Maxwell system in the gauge $A_{u}=0$ is ${ }^{9}$

$$
S=\lim _{u \rightarrow 0} \frac{N_{c}^{2} T^{2}}{16} \int \frac{d \omega d q}{(2 \pi)^{2}}\left[A_{t}^{\prime}(u, k) A_{t}(u,-k)-f(u) \boldsymbol{A}^{\prime}(u, k) \boldsymbol{A}(u,-k)\right]
$$

Using Maxwell's equations, the action can be written in terms of gauge-invariant variables as

$$
\begin{aligned}
S=\lim _{u \rightarrow 0} & \frac{N_{c}^{2} T^{2}}{16} \int \frac{d \omega d q}{(2 \pi)^{2}}\left[\frac{f(u)}{\mathfrak{q}^{2} f(u)-\mathfrak{w}^{2}} E_{z}^{\prime}(u, k) E_{z}(u,-k)\right. \\
& \left.-\frac{f(u)}{\mathfrak{w}^{2}}\left(E_{x}^{\prime}(u, k) E_{x}(u,-k)+E_{y}^{\prime}(u, k) E_{y}(u,-k)\right)\right]+ \text { contact terms }
\end{aligned}
$$

where "contact terms" do not contain derivatives of the electric fields. In order to find the correlation functions, one has to express the derivatives of the fields in terms of the boundary

\footnotetext{
${ }^{9}$ Normalization of the five-dimensional action $S=1 / 4 g_{B}^{2} \int \sqrt{-g} F_{A B} F^{A B}$ is fixed by $g_{B}^{2}=16 \pi^{2} R / N_{c}^{2}$ [24].
} 
values of the fields $A_{\mu}^{0}(k) \equiv A_{\mu}(u \rightarrow 0, k), E_{\mu}^{0}(k) \equiv E_{\mu}(u \rightarrow 0, k)$ using the solutions (4.7); applying Lorentzian AdS/CFT prescription [9], one finds ${ }^{10}$

$$
C_{\alpha \alpha}(\omega, q)=\frac{\delta^{2} S}{\delta A_{\alpha}^{0}(k) \delta A_{\alpha}^{0}(-k)}=\frac{\mathfrak{w}^{2} \delta^{2} S}{\delta E_{\alpha}^{0}(k) \delta E_{\alpha}^{0}(-k)}=-\frac{N_{c}^{2} T^{2} \mathcal{B}_{(\alpha)}}{8 \mathcal{A}_{(\alpha)}} .
$$

Similarly,

$$
\begin{aligned}
C_{t t}(\omega, q) & =\frac{N_{c}^{2} T^{2} \mathfrak{q}^{2} \mathcal{B}_{(z)}}{8\left(\mathfrak{q}^{2}-\mathfrak{w}^{2}\right) \mathcal{A}_{(z)}}, \\
C_{t z}(\omega, q) & =C_{z t}(\omega, q)=\frac{N_{c}^{2} T^{2} \mathfrak{w} \mathfrak{q} \mathcal{B}_{(z)}}{8\left(\mathfrak{q}^{2}-\mathfrak{w}^{2}\right) \mathcal{A}_{(z)}}, \\
C_{z z}(\omega, q) & =\frac{N_{c}^{2} T^{2} \mathfrak{w}^{2} \mathcal{B}_{(z)}}{8\left(\mathfrak{q}^{2}-\mathfrak{w}^{2}\right) \mathcal{A}_{(z)}} .
\end{aligned}
$$

Comparing these expressions with the general result (2.20), one finds

$$
\Pi^{T}(\omega, q)=-\frac{N_{c}^{2} T^{2} \mathcal{B}_{(\alpha)}(\omega, q)}{8 \mathcal{A}_{(\alpha)}(\omega, q)}, \quad \Pi^{L}(\omega, q)=-\frac{N_{c}^{2} T^{2} \mathcal{B}_{(z)}(\omega, q)}{8 \mathcal{A}_{(z)}(\omega, q)} .
$$

Thus the correlation functions are completely determined by the ratios of the connection coefficients of differential equations (4.5). In particular, poles of the correlators correspond to zeros of the coefficients $\mathcal{A}_{(\alpha)}(\mathfrak{w}, \mathfrak{q})$ and $\mathcal{A}_{(z)}(\mathfrak{w}, \mathfrak{q})$. To find the zeros, we impose Dirichlet boundary conditions on electric fields at $u=0$ for the solutions to equations (4.5) which satisfy the incoming wave conditions at the horizon. Physically, the horizon acts as a perfectly absorbing surface, while the boundary acts as a perfect conductor.

In order to determine the self-energies $\Pi^{T}(\omega, q), \Pi^{L}(\omega, q)$, one needs to know the solution to equations (4.5). Analytic solution is unknown, except for a special case of $\mathfrak{q}=0$, when the Dirichlet boundary value problem can be reformulated as a problem of solving a transcendental algebraic continued fraction equation $[11,26]$. For $\mathfrak{q}=0$, gauge-invariant variables $E_{i}$, $i=x, y, z$ obey the same equation ${ }^{11}$

$$
E_{i}^{\prime \prime}+\frac{f^{\prime}}{f} E_{i}^{\prime}+\frac{\mathfrak{w}^{2}}{u f^{2}} E_{i}=0
$$

The solution to the Dirichlet boundary value problem for Eq. (4.13) can be found exactly, and is given by Heun polynomials [11]. The quasinormal spectrum is

$$
\mathfrak{q}=0, \quad \mathfrak{w}=n(1-i), \quad n=0,1,2, \ldots .
$$

For $\mathfrak{q} \neq 0$, quasinormal spectra of perturbations $E_{\alpha}$ and $E_{z}$ (and, correspondingly, the poles of $\Pi^{T}(\omega, q)$ and $\left.\Pi^{L}(\omega, q)\right)$ differ from each other, and can be found numerically, as explained in Appendix B. A typical arrangement of quasinormal frequencies is shown in Fig. 目(the poles of $\Pi^{L}(\omega, q)$ were found numerically in [11]). Quasinormal frequencies are located symmetrically with respect to the imaginary axis, as is expected from the singularities of the corresponding correlation function in the dual field theory, see equation (2.10).

\footnotetext{
${ }^{10}$ See [9] for the definition of functional derivative in this context.

${ }^{11}$ As they should, in accord with rotation invariance in the dual field theory, see Eq. (2.18).
} 


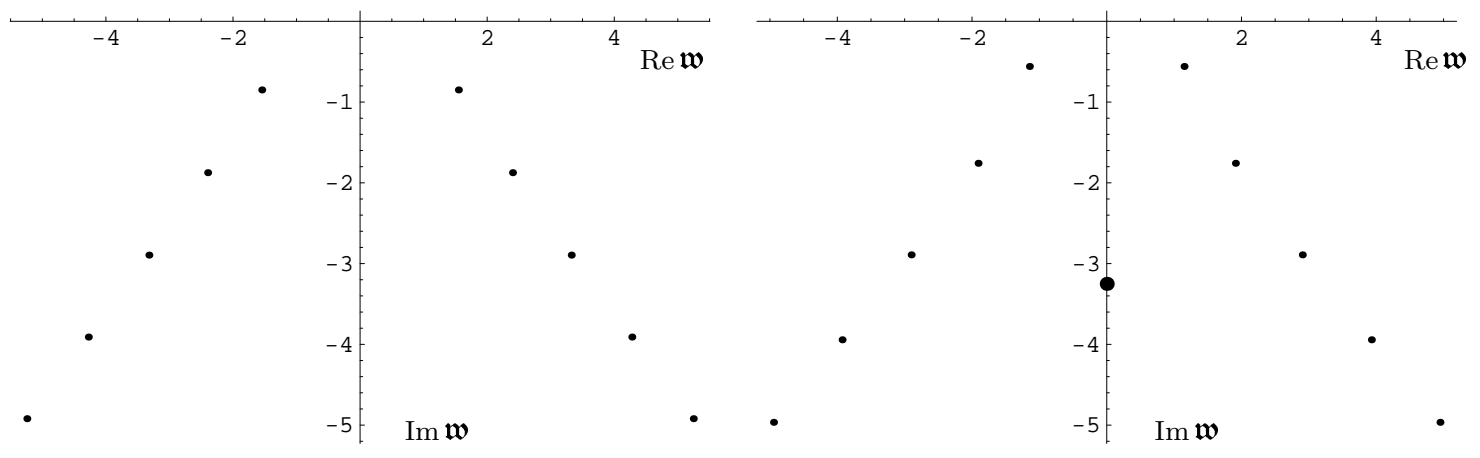

Figure 1: Quasinormal spectrum of electric field fluctuations in the plane of complex $\mathfrak{w} \equiv \omega / 2 \pi T$, shown for spatial momentum $\mathfrak{q} \equiv q / 2 \pi T=1$. Quasinormal frequencies on the left are defined by equation (4.5a) for the transverse electric field, and coincide with the poles of $\Pi^{T}(\omega, q)$, as explained in the text. Quasinormal frequencies on the right are defined by equation (4.5b) for the longitudinal electric field, and coincide with the poles of $\Pi^{L}(\omega, q)$. As $\mathfrak{q}$ decreases, all poles stay at a finite distance from the real axis, except for the one marked with a large dot. This pole is purely imaginary and approaches the origin in the limit $\mathfrak{q} \rightarrow 0$. The presence of this special quasinormal frequency is a manifestation of the diffusive relaxation of $R$-charge density fluctuations in the dual $\mathcal{N}=4 \mathrm{SYM}$ theory.

\section{Hydrodynamic approximation}

In the hydrodynamic limit one can find analytic solutions to equations (4.5) as a series in $\mathfrak{w} \ll 1, \mathfrak{q} \ll 1$. Assuming first that $\mathfrak{w}, \mathfrak{q}$ are of the same order, we get

$$
\begin{aligned}
& E_{\alpha}(u)=C_{\alpha} f(u)^{-i \mathfrak{w} / 2}\left[1+i \mathfrak{w} \log \frac{1+u}{2}+O\left(\mathfrak{w}^{2}, \mathfrak{q}^{2}\right)\right] \\
& E_{z}(u)=C_{z} f(u)^{-i \mathfrak{w} / 2}\left[1+i \mathfrak{w} \log \frac{1+u}{2}+\frac{i \mathfrak{q}^{2}}{\mathfrak{w}}(1-u)+O\left(\mathfrak{w}^{2}, \mathfrak{q}^{2}\right)\right],
\end{aligned}
$$

where $C_{\alpha}, C_{z}$ are normalization constants. Imposing Dirichlet boundary conditions at $u=0$, one finds that the equation $E_{\alpha}(0)=0$ has no solution compatible with the assumption $\mathfrak{w} \ll 1$. Holographic interpretation of this fact is that the function $\Pi^{T}(\omega, q)$ has no singularities in the hydrodynamic regime. The condition $E_{z}(0)=0$ leads to $\mathfrak{w}=-i \mathfrak{q}^{2}+O\left(\mathfrak{q}^{3}\right)$. This is the lowest hydrodynamic quasinormal frequency of the Dirichlet boundary value problem for $E_{z}$. Accordingly, in the hydrodynamic regime function $\Pi^{L}(\omega, q)$ has a pole at ${ }^{12}$

$$
\omega=-i D_{\mathrm{R}} q^{2}
$$

where $D_{\mathrm{R}}=1 / 2 \pi T$. Physically, it corresponds to the R-charge diffusion with the diffusion constant $D_{\mathrm{R}}$. By comparing the solutions (4.15) to the definition of connection coefficients

${ }^{12}$ One may question the validity of this result since it implies that $\mathfrak{w} \sim \mathfrak{q}^{2}$, whereas the solution 4.15b was obtained under the assumption that $\mathfrak{w}$ and $\mathfrak{q}$ are of the same order. To check our result, we introduce a new parameter $\mu=\mathfrak{w} / \mathfrak{q} \sim \mathfrak{q}$. Solving Eq. (4.5b) perturbatively in $\mu \ll 1, \mathfrak{q} \ll 1$, where $\mu$ and $\mathfrak{q}$ are of the same order, we find $E_{z}(u)=C_{z} f^{-i \mu \mathfrak{q} / 2}[1+i \mathfrak{q}(1-u) / \mu+O(\mu)]$. The condition $E_{z}(0)=0$ again gives the dispersion relation $\mathfrak{w}=-i \mathfrak{q}^{2}$. 
(4.7), one finds $\mathcal{A}_{(\alpha)}=1, \mathcal{B}_{(\alpha)}=i \mathfrak{w}, \mathcal{A}_{(z)}=1+i \mathfrak{q}^{2} / \mathfrak{w}, \mathcal{B}_{(z)}=i \mathfrak{w}-i \mathfrak{q}^{2} / \mathfrak{w}$. Substituting these connection coefficients into AdS/CFT results (4.10) and (4.11), one reproduces the $R$-current correlators in the low-frequency approximation found earlier in [15].

\subsection{Stress-energy tensor correlators}

Correlation functions of stress-energy tensor in the finite temperature $\mathcal{N}=4 \mathrm{SYM}$ at large $N_{c}$ and strong coupling were studied in $[11,15,16]$. Here we use the gauge-invariant variables approach to identify the correct associated boundary value problem, and to obtain new results for the correlators in the sound wave channel.

To compute correlators of the stress-energy tensor in AdS/CFT correspondence, one considers metric fluctuations $h_{A B}$ of the supergravity background. To linear order in $h_{A B}$, the Einstein equations are

$$
\mathcal{R}_{A B}^{(1)}=-\frac{4}{R^{2}} h_{A B}
$$

where $\mathcal{R}_{A B}^{(1)}$ is the linearized Ricci tensor evaluated in the background (4.2). Translation invariance for the $t, x, y, z$ coordinates implies that metric perturbations can be Fourier transformed, and classified according to their transformations with respect to the rotation group $O(2)$, as discussed in Section 3.2. As we shall see shortly, quasinormal spectra of the three gaugeinvariant variables $(3.11)-(3.13)$ appear correspondingly as the poles of the three functions $G_{1}, G_{2}, G_{3}$ in Eq. 2.25) which determines the two-point correlation function of stress-energy tensor in a scale-invariant theory.

\subsubsection{Scalar channel}

According to the discussion in Section 3, the equation satisfied by the component $h_{x y}$ of the perturbed metric decouples from the rest of Einstein equations. The gauge-invariant function $Z_{3}=H_{x y}=h_{y}^{x}$ satisfies the equation for a minimally coupled massless scalar in the background (4.2),

$$
Z_{3}^{\prime \prime}-\frac{1+u^{2}}{u f} Z_{3}^{\prime}+\frac{\mathfrak{w}^{2}-\mathfrak{q}^{2} f}{u f^{2}} Z_{3}=0
$$

The exponents of this equation near $u=0$ are 0 and 2 , therefore asymptotic behavior of $Z_{3}$ near the boundary is

$$
Z_{3}(u)=\mathcal{A}_{(3)}(1+\cdots)+\mathcal{B}_{(3)} u^{2}+\cdots,
$$

where ellipses denote higher powers of $u$. The relevant part of the boundary gravitational action (Eq.(6.9) of [15]) can be written using our notations as

$$
S=-\frac{\pi^{2} N_{c}^{2} T^{4}}{8} \lim _{u \rightarrow 0} \int \frac{d \omega d q}{(2 \pi)^{2}} \frac{f(u)}{u} Z_{3}^{\prime}(u, k) Z_{3}(u,-k) .
$$

Proceeding as in Section 4.1, we obtain the correlator ${ }^{13}$

$$
G_{x y, x y}=-\frac{\pi^{2} N_{c}^{2} T^{4} \mathcal{B}_{(3)}}{2 \mathcal{A}_{(3)}}
$$

\footnotetext{
${ }^{13}$ Terms analytic in $\mathfrak{w}$ and $\mathfrak{q}$ are ignored, even if they are divergent as $u \rightarrow 0$.
} 


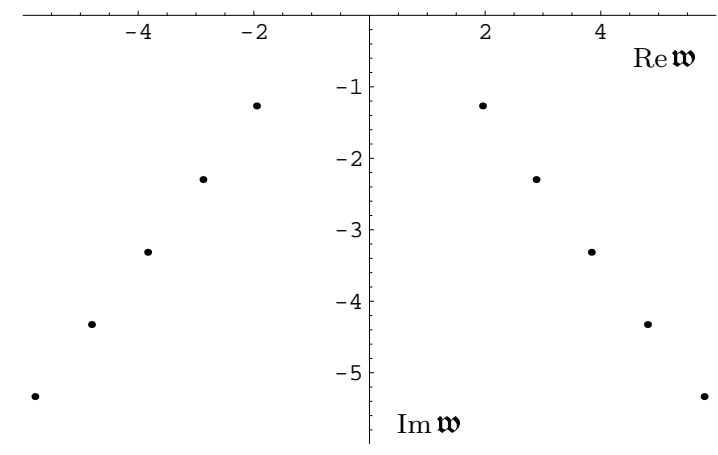

Figure 2: Quasinormal spectrum of gravitational fluctuations in the scalar channel, shown in the plane of complex $\mathfrak{w} \equiv \omega / 2 \pi T$, for spatial momentum $\mathfrak{q} \equiv q / 2 \pi T=1$. The quasinormal frequencies coincide with poles of $G_{3}(\omega, q)$, as explained in the text. As $\mathfrak{q} \rightarrow 0$, all poles stay at a finite distance from the real axis, as one expects from the absence of hydrodynamic singularities in $G_{3}(\omega, q)$.

Comparing this expression to the general result (2.34), we find

$$
G_{3}(\omega, q)=-\frac{\pi^{2} N_{c}^{2} T^{4} \mathcal{B}_{(3)}(\omega, q)}{\mathcal{A}_{(3)}(\omega, q)} .
$$

Turning now to the connection between quasinormal spectrum and AdS/CFT correlators, we see that the condition $\mathcal{A}_{(3)}(\mathfrak{w}, \mathfrak{q})=0$ determines the singularities of $G_{3}$. From the definition of connection coefficients (4.19) it is evident that the condition $\mathcal{A}_{(3)}(\mathfrak{w}, \mathfrak{q})=0$ is equivalent to imposing the Dirichlet boundary condition on fluctuations, $Z_{3}(u=0)=0$.

The quasinormal spectrum of fluctuations obeying incoming wave boundary condition at the horizon and Dirichlet condition $Z_{3}(u=0)=0$ at the boundary was numerically computed in $[11,26]$. The spectrum $\mathfrak{w}_{n}$ is discrete, (presumably) infinite, and almost equidistant. For $\mathfrak{q}=0$ its asymptotics for higher modes is well approximated by a simple formula ${ }^{14}[26]$

$$
\omega_{n}=2 \pi \operatorname{Tn}( \pm 1-i), \quad n \rightarrow \infty .
$$

A typical arrangement of quasinormal frequencies is shown in Fig. 2. Quasinormal frequencies are located symmetrically with respect to the imaginary axis, as is expected from the singularities of the corresponding correlation function in the dual field theory, see Eq. (2.11).

\section{Hydrodynamic approximation}

In the hydrodynamic limit $\mathfrak{w} \ll 1, \mathfrak{q} \ll 1$, analytic solution to Eq. (4.18) can be found,

$$
Z_{3}(u)=C_{3} f(u)^{-i \mathfrak{w} / 2}\left[1+O\left(\mathfrak{w}^{2}, \mathfrak{q}^{2}\right)\right],
$$

where $C_{3}$ is a normalization constant. By comparing this solution with the definition of connection coefficients (4.19), one finds $\mathcal{A}_{(3)}=1+O\left(\mathfrak{w}^{2}\right), \mathcal{B}_{(3)}=i \mathfrak{w} / 2+O\left(\mathfrak{w} \mathfrak{w}^{2}, \mathfrak{q}^{2}, \mathfrak{w} \mathfrak{q}\right)$.

\footnotetext{
${ }^{14}$ For an analytic approach to the asymptotic behavior (4.23) see [27].
} 
Substituting these connection coefficients into the AdS/CFT result (4.21), one reproduces the correlator of transverse components of stress tensor in the low-frequency approximation found earlier in [15]. The equation $\mathcal{A}_{(3)}(\mathfrak{w}, \mathfrak{q})=0$ does not have solutions compatible with the condition $\mathfrak{w} \ll 1$. This is consistent with our expectations in Section that $G_{3}$ has no hydrodynamic singularities.

\subsubsection{Shear channel}

According to the discussion in Section 3, equations satisfied by the components $h_{t x}, h_{z x}$, and $h_{u x}$ of the perturbed metric form a closed set. In the radial gauge $h_{u A}=0$, they read

$$
\begin{aligned}
& H_{z x}^{\prime}=-\frac{\mathfrak{w}}{\mathfrak{q} f} H_{t x}^{\prime}, \\
& H_{t x}^{\prime \prime}=\frac{1}{u} H_{t x}^{\prime}+\frac{\mathfrak{w} \mathfrak{q}}{u f} H_{z x}+\frac{\mathfrak{q}^{2}}{u f} H_{t x},
\end{aligned}
$$

where $H_{t x}=u h_{t x} /(\pi T R)^{2}, H_{z x}=u h_{z x} /(\pi T R)^{2}$. Using these equations, one finds that $Z_{1}(u) \equiv \mathfrak{q} H_{t x}(u)+\mathfrak{w} H_{z x}(u)$ satisfies the following second-order ODE

$$
Z_{1}^{\prime \prime}+\frac{\left(\mathfrak{w}^{2}-\mathfrak{q}^{2} f\right) f-u \mathfrak{w}^{2} f^{\prime}}{u f\left(\mathfrak{q}^{2} f-\mathfrak{w}^{2}\right)} Z_{1}^{\prime}+\frac{\mathfrak{w}^{2}-\mathfrak{q}^{2} f}{u f^{2}} Z_{1}=0 .
$$

The exponents of Eq. (4.26) at $u=0$ are 0 and 2, and thus asymptotic behavior of $Z_{1}$ near the boundary is

$$
Z_{1}(u)=\mathcal{A}_{(1)}(1+\ldots)+\mathcal{B}_{(1)} u^{2}+\ldots
$$

where ellipses denote higher powers of $u$. The relevant part of the boundary gravitational action is given by Eq. (6.19) of [15]. Expressed in terms of the gauge-invariant variable $Z_{1}$, the action is

$$
S=-\frac{\pi^{2} N_{c}^{2} T^{4}}{8} \lim _{u \rightarrow 0} \int \frac{d \omega d q}{(2 \pi)^{2}} \frac{f(u)}{u\left(\mathfrak{w}^{2}-\mathfrak{q}^{2} f(u)\right)} Z_{1}^{\prime}(u, k) Z_{1}(u,-k)+\text { contact terms } .
$$

Proceeding as in Section 4.1, we find after comparing the expression for the correlator with (2.28) - 2.30)

$$
G_{1}(\omega, q)=-\frac{\pi^{2} N_{c}^{2} T^{4} \mathcal{B}_{(1)}(\omega, q)}{\mathcal{A}_{(1)}(\omega, q)} .
$$

Again, the condition $\mathcal{A}_{(1)}(\omega, q)=0$ is equivalent to Dirichlet boundary condition $Z_{1}(u=0)=$ 0. A typical arrangement of quasinormal frequencies is shown in Fig. 3 .

\section{Hydrodynamic approximation}

In the limit $\mathfrak{w} \ll 1, \mathfrak{q} \ll 1$, the perturbative analytical solution to Eq. (4.26) satisfying the incoming wave boundary condition at the horizon is ${ }^{15}$

$$
Z_{1}(u)=C_{1} f(u)^{-i \mathfrak{w} / 2}\left(1+\frac{i \mathfrak{q}^{2} f}{2 \mathfrak{w}}+O\left(\mathfrak{w}^{2}, \mathfrak{q}^{2}, \mathfrak{w} \mathfrak{q}\right)\right)
$$

\footnotetext{
${ }^{15}$ The argument of footnote 12 regarding the scaling of $\mathfrak{w}, \mathfrak{q}$ also applies here. The boundary condition constrains $\mathfrak{w}$ and $\mathfrak{q}$ in such a way that the initial assumption that $\mathfrak{w}$ and $\mathfrak{q}$ are of the same order is invalid. Using the correct scaling we find that the result (4.32) remains unchanged.
} 


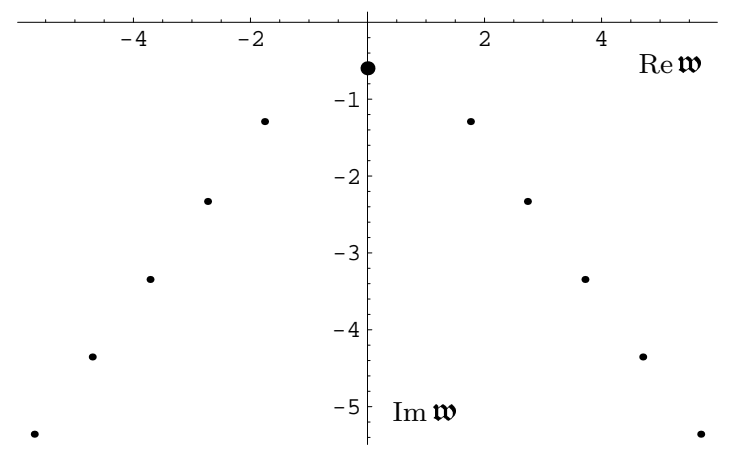

Figure 3: Quasinormal spectrum of gravitational fluctuations in the shear channel, shown in the plane of complex $\mathfrak{w} \equiv \omega / 2 \pi T$, for spatial momentum $\mathfrak{q} \equiv q / 2 \pi T=1$. The quasinormal frequencies coincide with poles of $G_{1}(\omega, q)$, as explained in the text. As $\mathfrak{q}$ decreases, all poles stay at a finite distance away from the real axis, except for the one marked with a large dot. This pole is purely imaginary and approaches the origin in the limit $\mathfrak{q} \rightarrow 0$. The presence of this special quasinormal frequency is a manifestation of the diffusive relaxation of transverse momentum density fluctuations in the dual $\mathcal{N}=4$ SYM theory.

where $C_{1}$ is a normalization constant. Expanding for small $u$, we find the connection coefficients

$$
\mathcal{A}_{(1)}=1+\frac{i \mathfrak{q}^{2}}{2 \mathfrak{w}}+O\left(\mathfrak{w}^{2}, \mathfrak{q}^{2}, \mathfrak{w} \mathfrak{q}\right), \quad \mathcal{B}_{(1)}=\frac{i\left(\mathfrak{w}^{2}-\mathfrak{q}^{2}\right)}{2 \mathfrak{w}}+O\left(\mathfrak{w}^{2}, \mathfrak{q}^{2}, \mathfrak{w} \mathfrak{q}\right) .
$$

The Dirichlet condition $Z_{1}(u=0)=0$ gives the hydrodynamic quasinormal frequency $\mathfrak{w}=$ $-i \mathfrak{q}^{2} / 2+O\left(\mathfrak{q}^{3}\right)$. It is interpreted as the dispersion relation for the shear mode,

$$
\omega=-i \gamma_{\eta} q^{2}+O\left(q^{3}\right),
$$

where $\gamma_{\eta}=1 / 4 \pi T$. For the function $G_{1}(\omega, q)$ in this approximation we find

$$
G_{1}(\omega, q)=\frac{\pi N_{c}^{2} T^{3}\left(\omega^{2}-q^{2}\right)}{4\left(i \omega-q^{2} / 4 \pi T\right)}
$$

in agreement with the result obtained earlier in [15]. The quasinormal spectrum for frequencies beyond the hydrodynamic limit was obtained in [11] using a slightly different approach.

\subsubsection{Sound channel}

According to the discussion in Section 3, equations obeyed by the components of the metric $H_{t t}=u h_{t t} / f(\pi T R)^{2}, H_{t z}=u h_{t z} /(\pi T R)^{2}, H_{z z}=u h_{z z} /(\pi T R)^{2}, H_{a a}=u\left(h_{x x}+h_{y y}\right) /(\pi T R)^{2}$ form a closed system of equations (in the radial gauge $\left.h_{u A}=0\right)$. These equations are lengthy, and we present them in Appendix A. Using the equations of motion (A.1) - (A.4) one can show that the gauge-invariant combination

$$
Z_{2}(u) \equiv 4 \mathfrak{w} \mathfrak{q} H_{t z}+2 \mathfrak{w}^{2} H_{z z}+H_{a a}\left[\mathfrak{q}^{2}(2-f)-\mathfrak{w}^{2}\right]+2 \mathfrak{q}^{2} f H_{t t}
$$


obeys the following second-order differential equation:

$$
\begin{aligned}
Z_{2}^{\prime \prime} & -\frac{3 \mathfrak{w}^{2}\left(1+u^{2}\right)+\mathfrak{q}^{2}\left(2 u^{2}-3 u^{4}-3\right)}{u f\left(3 \mathfrak{w}^{2}+\mathfrak{q}^{2}\left(u^{2}-3\right)\right)} Z_{2}^{\prime} \\
& +\frac{3 \mathfrak{w}^{4}+\mathfrak{q}^{4}\left(3-4 u^{2}+u^{4}\right)+\mathfrak{q}^{2}\left(4 u^{5}-4 u^{3}+4 u^{2} \mathfrak{w}^{2}-6 \mathfrak{w}^{2}\right)}{u f^{2}\left(3 \mathfrak{w}^{2}+\mathfrak{q}^{2}\left(u^{2}-3\right)\right)} Z_{2}=0 .
\end{aligned}
$$

In the limit $u \rightarrow 0$ this equation coincides with Eq. (4.18) obeyed by a minimally coupled massless scalar, and thus the behavior of the solution $Z_{2}(u)$ near the boundary is given by

$$
Z_{2}(u)=\mathcal{A}_{(2)}(1+\cdots)+\mathcal{B}_{(2)} u^{2}+\cdots,
$$

where ellipses denote higher powers of $u$. Using the equations of motion (A.1) - (A.4), the relevant part of the on-shell boundary gravitational action (quadratic in fluctuations) can be written as ${ }^{16}$

$$
S_{B}^{(2)}=\lim _{u \rightarrow 0} \int \frac{d \omega d q}{(2 \pi)^{2}} A(\mathfrak{w}, \mathfrak{q}, u) Z_{2}^{\prime}(u, k) Z_{2}(u,-k)+S_{C T}^{(2)},
$$

where

$$
A(\mathfrak{w}, \mathfrak{q}, u)=\frac{3 N_{c}^{2} \pi^{2} T^{4} f(u)}{32 u\left(3 \mathfrak{w}^{2}-\mathfrak{q}^{2}\left(3-u^{2}\right)\right)^{2}},
$$

and the "contact term" part $S_{C T}^{(2)}$ does not contain derivatives of the fluctuations (its boundary value is given in Appendix A). In order to compute the stress-tensor correlation functions, we need to solve the "wave equation" (4.35) for $Z_{2}(u)$ subject to the boundary condition

$$
Z_{2}(u=0)=4 \mathfrak{w} \mathfrak{q} H_{t z}^{0}+2 \mathfrak{w}^{2} H_{z z}^{0}+H_{a a}^{0}\left(\mathfrak{q}^{2}-\mathfrak{w}^{2}\right)+2 \mathfrak{q}^{2} H_{t t}^{0},
$$

substitute the result into the action (4.37) and take the appropriate functional derivatives ${ }^{17}$ with respect to the boundary values of the fields $H_{t t}^{0}, H_{t z}^{0}, H_{z z}^{0}, H_{a a}^{0}$. For example, for the correlator $G_{t t, t t}$ we have

$$
G_{t t, t t}=-4 \frac{\delta^{2} S_{B}^{(2)}}{\delta H_{t t}^{0}(k) \delta H_{t t}^{0}(-k)} .
$$

Using the expansion (4.36), we find that the correlators are given by Eqs. (2.31) - (2.33) with $^{18}$

$$
G_{2}(\omega, q)=-\frac{N_{c}^{2} \pi^{2} T^{4} \mathcal{B}_{(2)}(\omega, q)}{\mathcal{A}_{(2)}(\omega, q)}+\text { contact terms } .
$$

The problem of computing correlation functions in the dual theory is thus reduced to finding the connection coefficients $\mathcal{A}_{(2)}$ and $\mathcal{B}_{(2)}$ of the second order ODE $(4.35)$. Zeroes of $\mathcal{A}_{(2)}(\mathfrak{w}, \mathfrak{q})$

\footnotetext{
${ }^{16}$ The action involving the relevant components of the metric is written in Appendix A. In order to find $A(\mathfrak{w}, \mathfrak{q}, u)$, we form the difference $S_{B}^{(2)}-\int d \omega d q /(2 \pi)^{2} A(\mathfrak{w}, \mathfrak{q}, u) Z_{2}^{\prime}(u, k) Z_{2}(u,-k)$, then use the equations of motion A.1 - A.4 to eliminate all derivatives in the difference except $H_{t t}^{\prime}$, and find $A(\mathfrak{w}, \mathfrak{q}, u)$ by requiring that the coefficient in front of $H_{t t}^{\prime}$ should vanish.

${ }^{17}$ For correct normalization of the coupling between the boundary gravitational fluctuations and the stressenergy tensor, see Eq. (3.18) of [16].

${ }^{18}$ Terms analytic in $\mathfrak{w}$ and $\mathfrak{q}$ are ignored, even if they are divergent as $u \rightarrow 0$.
} 


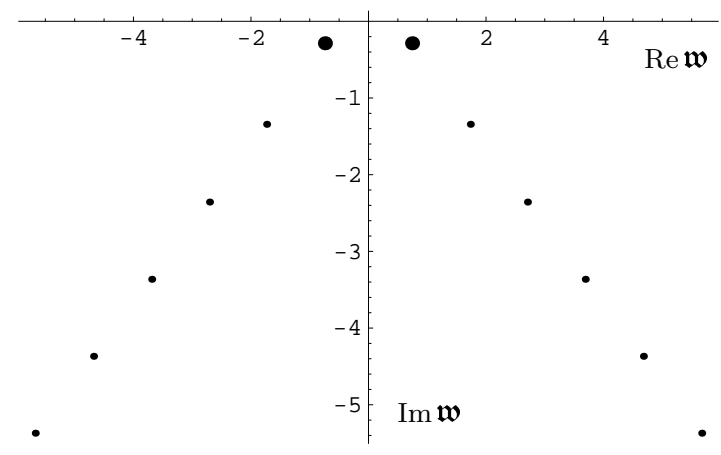

Figure 4: Quasinormal spectrum of gravitational fluctuations in the sound channel, shown in the plane of complex $\mathfrak{w} \equiv \omega / 2 \pi T$, for spatial momentum $\mathfrak{q} \equiv q / 2 \pi T=1$. The quasinormal frequencies coincide with the poles of $G_{2}(\omega, q)$, as explained in the text. As $\mathfrak{q}$ decreases, all poles stay at a finite distance away from the real axis, except for the ones marked with large dots, which approach the origin as $\mathfrak{q} \rightarrow 0$ (see Appendix B for the corresponding dispersion curves). Such behavior of the lowest quasinormal frequencies is a manifestation of oscillatory relaxation of longitudinal momentum density (as well as energy density) fluctuations in the dual $\mathcal{N}=4$ SYM theory.

appear as poles of the correlators. Finding the poles is therefore equivalent to solving the boundary value problem for the gauge invariant variable $Z_{2}(u)$ obeying the incoming wave boundary condition at the horizon $u=1$ and Dirichlet condition $Z_{2}(u=0)=0$ at the boundary.

For $\mathfrak{q}=0$, eqs. (4.18), (4.26), (4.35) all reduce to the equation for a minimally coupled massless scalar at zero spatial momentum, and consequently all have the same quasinormal spectrum with the asymptotics (4.23). This is expected, in accord with rotation invariance in the dual field theory, see Eq. $(2.26)$. For $\mathfrak{q} \neq 0$, the spectrum can be found numerically, as explained in Appendix B; a typical arrangement of quasinormal frequencies is shown in Fig. 4 . However, for small momenta, the lowest quasinormal frequency can be found analytically.

\section{Hydrodynamic approximation}

In the hydrodynamic regime $\mathfrak{w} \ll 1, \mathfrak{q} \ll 1$, Eq. (4.35) for $Z_{2}(u)$ can be solved perturbatively in $\mathfrak{w}$ and $\mathfrak{q}$. Introducing the book-keeping parameter $\lambda$, rescaling $\mathfrak{w} \rightarrow \lambda \mathfrak{w}, \mathfrak{q} \rightarrow \lambda \mathfrak{q}$, and expanding in $\lambda \ll 1$, to first order in $\lambda$ we find

$$
Z_{2}(u)=C_{2} f(u)^{-i \mathfrak{w} / 2}\left[\frac{\mathfrak{q}^{2}\left(1+u^{2}\right)-3 \mathfrak{w}^{2}}{4 \mathfrak{q}^{2}}-\frac{i \mathfrak{w} f(u)}{2}\right]
$$

where $C_{2}$ is a normalization constant. Imposing Dirichlet boundary condition $Z_{2}(u=0)=0$ gives the lowest $(|\mathfrak{w}| \ll 1)$ overtone of the quasinormal spectrum

$$
\mathfrak{w}= \pm \frac{\mathfrak{q}}{\sqrt{3}}-\frac{i \mathfrak{q}^{2}}{3}+O\left(\mathfrak{q}^{3}\right)
$$



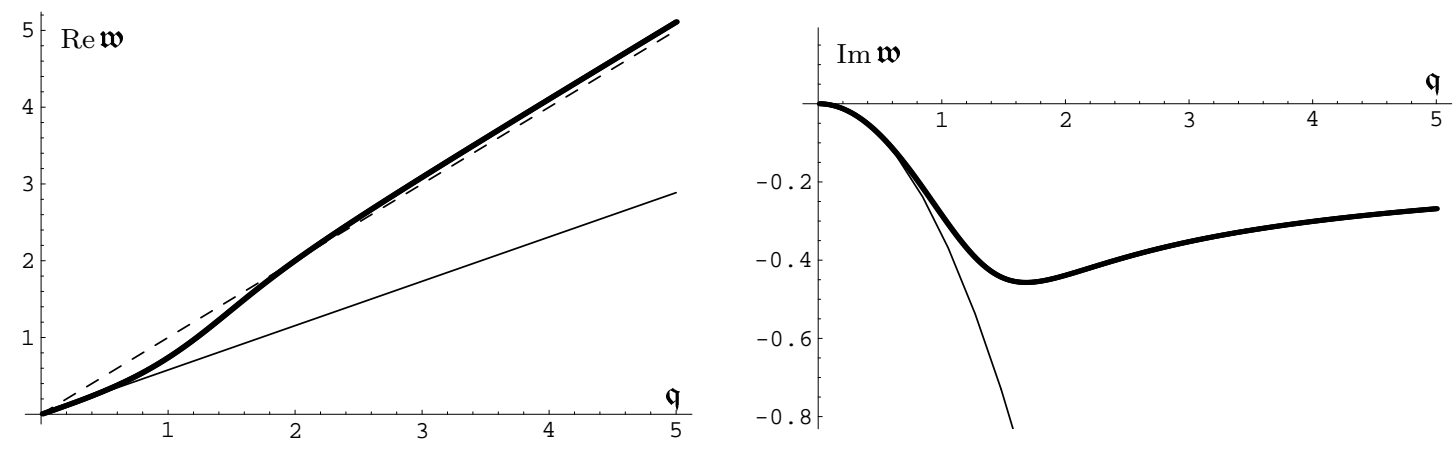

Figure 5: Real and imaginary parts of the lowest quasinormal (sound wave) frequency as a function of spatial momentum. Light curves correspond to the analytic approximation (4.43) for small $\mathfrak{q}$. Dashed line is $\mathfrak{w}=\mathfrak{q}$. As $\mathfrak{q}$ grows, the dispersion curve enters the region where $\partial \operatorname{Re}(\mathfrak{w}) / \partial \mathfrak{q}>1$. This, however, happens for $\mathfrak{q} \sim 1$, where the corresponding singularity of the energy-momentum correlation function no longer has the interpretation of a sound wave.

In the holographically dual finite temperature quantum field theory, Eq. (4.43) appears as a pole in the retarded correlator of stress-energy tensors, and is interpreted as dispersion relation for the sound wave mode,

$$
\omega(q)= \pm v_{s} q-i \Gamma_{s} q^{2}+O\left(q^{3}\right)
$$

The values for the speed of sound $v_{s}=1 / \sqrt{3}$ and the attenuation constant $\Gamma_{s}=1 / 6 \pi T$ coincide with those found previously in [16]. Expanding the solution (4.42) near $u=0$ and comparing with $(4.19)$, we identify the coefficients $\mathcal{A}_{(2)}=\left(\mathfrak{q}^{2}-3 \mathfrak{w} \mathfrak{w}^{2}-2 i \mathfrak{w} \mathfrak{q}^{2}\right) / 4 \mathfrak{q}^{2}, \mathcal{B}_{(2)}=$ $\left(2 \mathfrak{q}^{2}+5 i \mathfrak{w} \mathfrak{q}^{2}-3 i \mathfrak{w}^{3}\right) / 8 \mathfrak{q}^{2}$. Thus to leading order in the hydrodynamic approximation we obtain

$$
G_{2}(\omega, q)=\frac{N_{c}^{2} \pi^{2} T^{4} q^{2}}{3 \omega^{2}-q^{2}}
$$

The dispersion relation $\mathfrak{w}=\mathfrak{w}(\mathfrak{q})$ for the sound wave frequency can be determined numerically

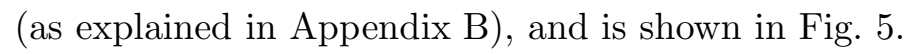

\section{Discussion}

In this paper, we proposed a general approach for identifying quasinormal spectra of asymptotically AdS spacetimes with the poles of the retarded correlators in the holographically dual finite temperature field theory. Our demonstration in Section 1 that quasi-normal spectrum of gauge-invariant perturbations in asymptotically AdS spacetimes has a precise interpretation in dual field theory was specific to the case of five-dimensional AdS-Schwarzschild background with a plane-symmetric event horizon. Although we expect the same to be true in a more general setting as discussed in Section 3 (such as black holes rather than branes, or more complicated backgrounds), we did not give a general proof. Explicit computations for other backgrounds can be done along the lines of Section 4. 
From the dual field theory point of view, a noteworthy observation is that the only singularities of thermal Green's functions identified in the supergravity approach are simple poles. It may indeed be true that real-time thermal correlation functions of gauge-invariant operators are meromorphic in complex frequency plane; we leave the analysis of analytic structure for future investigation. Of course, this simple nature of singularities is possible only at infinite $N_{c}$; for example, it is known that low-energy correlation functions of conserved currents develop branch cuts in the complex frequency plane, with discontinuities across the cuts suppressed in the $N_{c} \rightarrow \infty$ limit (such cuts are not visible in classical supergravity and reflect quantum modifications of the quasinormal spectrum) [28]. In a sense, the situation is similar to zero-temperature spectrum of confining gauge theories, when resonances become stable as $N_{c} \rightarrow \infty$ [29]. However, at finite temperature, the poles of the real-time correlation functions can not be automatically interpreted as quasi-particles propagating in thermal bath - a definite interpretation can be given only after the full spectral density (not just the poles) is known. In infinitely strongly coupled $\mathcal{N}=4$ SYM theory, the quasiparticle interpretation is unlikely because of the unique energy scale (temperature). On the other hand, it is possible that $\alpha^{\prime}$ corrections to the quasinormal spectrum will reveal new poles corresponding to heavy excitations (whose mass scales with the 't Hooft coupling of the dual field theory) which can be interpreted as quasiparticles.

\section{Acknowledgments}

We would like to thank D. T. Son and L. G. Yaffe for helpful conversations, C. P. Herzog for comments on the manuscript, and J. Mas for correspondence and comments on the paper [16]. A. O. S. would like to thank the organizers of the "QCD and String Theory" workshop at the KITP, UC Santa Barbara, where part of this work was completed. The work of P. K. K. was supported in part by the National Science Foundation under Grant No. PHY99-07949. Research at Perimeter Institute is supported in part by funds from NSERC of Canada. 


\section{A. Equations of motion and boundary action for the sound channel}

Fluctuations of the sound wave mode satisfy a system of differential equations

$$
\begin{aligned}
H_{t z}^{\prime} & =\frac{2 \mathfrak{w} \mathfrak{q}}{f+2}\left(H_{a a}-H_{t t}\right)+\frac{\left(u^{3}-3 u-2 \mathfrak{w}^{2}\right)}{\mathfrak{q} f(f+2)}\left(\mathfrak{w} H_{a a}+\mathfrak{w} H_{z z}+2 \mathfrak{q} H_{t z}\right) \\
& -\frac{3 \mathfrak{w} f}{\mathfrak{q}(f+2)} H_{t t}^{\prime}, \\
H_{a a}^{\prime} & =\frac{\mathfrak{w}\left(u^{3}-3 u-2 \mathfrak{w}^{2}\right)}{\mathfrak{q}^{2} f^{2}(f+2)}\left(\mathfrak{w} H_{a a}+\mathfrak{w} H_{z z}+2 \mathfrak{q} H_{t z}\right)-\frac{3 \mathfrak{w}^{2}-\mathfrak{q}^{2}(f+2)}{\mathfrak{q}^{2}(f+2)} H_{t t}^{\prime} \\
& +\frac{2 \mathfrak{w}^{2}}{f(f+2)} H_{a a}+\frac{u^{3}-3 u-2 \mathfrak{w}^{2}}{f(f+2)} H_{t t}, \\
H_{z z}^{\prime} & =\frac{2}{f(f+2)}\left[\mathfrak{w}^{2} H_{a a}+\mathfrak{w}^{2} H_{z z}+\mathfrak{q}^{2} f\left(H_{t t}-H_{a a}\right)+2 \mathfrak{w} \mathfrak{q} H_{t z}\right]+\frac{3 f H_{t t}^{\prime}}{f+2} \\
& +\frac{1}{f(f+2)}\left[2 \mathfrak{w}^{2} H_{a a}+\left(u^{3}-3 u-2 \mathfrak{w}^{2}\right) H_{t t}\right]-\frac{3 \mathfrak{w}^{2}-\mathfrak{q}^{2}(f+2)}{\mathfrak{q}^{2}(f+2)} H_{t t}^{\prime} \\
& +\frac{\mathfrak{w}\left(u^{3}-3 u-2 \mathfrak{w}^{2}\right)}{\mathfrak{q}^{2} f^{2}(f+2)}\left(\mathfrak{w} H_{a a}+\mathfrak{w} H_{z z}+2 \mathfrak{q} H_{t z}\right), \\
H_{t t}^{\prime \prime} & =\frac{1}{2 u^{2} f^{2}(f+2)}\left\{6 u f\left(1+u^{2}\right) H_{t t}^{\prime}+2 u f\left[2 \mathfrak{w}^{2}+\mathfrak{q}^{2}\left(1+u^{2}\right)\right] H_{a a}+4 \mathfrak{w}^{2} u f H_{z z}\right. \\
& \left.+8 \mathfrak{w} \mathfrak{q} u f H_{t z}+4 u f^{2} \mathfrak{q}^{2} H_{t t}\right\} .
\end{aligned}
$$

The part of the boundary action quadratic in fluctuations is [16]

$$
\begin{gathered}
S_{B}^{(2)}=\lim _{u \rightarrow 0} \frac{\pi^{2} N_{c}^{2} T^{4}}{8} \int d^{4} x\left[\frac{1}{8}\left(3 H_{t t}^{2}-12 H_{t z}^{2}+2 H_{t t} H_{i i}+2 H_{z z} H_{a a}-H_{z z}^{2}\right)\right. \\
\left.-\frac{f(u)}{2 u}\left(H_{t z}^{2}+\frac{1}{4} H_{a a}^{2}-H_{t t} H_{i i}+H_{z z} H_{a a}\right)^{\prime}\right]
\end{gathered}
$$

where prime denotes the derivative with respect to $u$, and expressions such as $H_{t t}^{2}$ are to be understood as $H_{t t}(u, k) H_{t t}(u,-k)$. The boundary value of the "contact term" part of the gravitational action (4.37) is given by

$$
\begin{aligned}
S_{C T}^{(2)}(0) & =\lim _{u \rightarrow 0} S_{C T}^{(2)}=-\frac{N_{c}^{2} \pi^{2} T^{4}}{48}\left[\left(H_{a a}^{0}\right)^{2}-\frac{\mathfrak{q}^{2}+3 \mathfrak{w}^{2}}{2\left(\mathfrak{q}^{2}-\mathfrak{w}^{2}\right)} H_{a a}^{0} H_{t t}^{0}-\frac{4 \mathfrak{w} \mathfrak{q}}{\mathfrak{q}^{2}-\mathfrak{w}^{2}} H_{a a}^{0} H_{t z}^{0}\right. \\
& -\frac{\left.29 \mathfrak{q}^{4}-30 \mathfrak{w}^{2} \mathfrak{q}^{2}+9 \mathfrak{w} \mathfrak{w}^{4}\right)}{4\left(\mathfrak{q}^{2}-\mathfrak{w}^{2}\right)^{2}}\left(H_{t t}^{0}\right)^{2}-\frac{4 \mathfrak{w} \mathfrak{q}\left(5 \mathfrak{q}^{2}-3 \mathfrak{w}^{2}\right)}{\left(\mathfrak{q}^{2}-\mathfrak{w}^{2}\right)^{2}} H_{t t}^{0} H_{t z}^{0} \\
& -\frac{3 \mathfrak{q}^{2}+\mathfrak{w}^{2}}{2\left(\mathfrak{q}^{2}-\mathfrak{w}^{2}\right)} H_{a a}^{0} H_{z z}^{0}-\frac{9 \mathfrak{q}^{4}+2 \mathfrak{w}^{2} \mathfrak{q}^{2}-3 \mathfrak{w}^{4}}{2\left(\mathfrak{q}^{2}-\mathfrak{w}^{2}\right)^{2}} H_{t t}^{0} H_{z z}^{0}-\frac{4 \mathfrak{w} \mathfrak{q}\left(3 \mathfrak{q}^{2}-\mathfrak{w}^{2}\right)}{\left(\mathfrak{q}^{2}-\mathfrak{w}^{2}\right)^{2}} H_{t z}^{0} H_{z z}^{0} \\
& \left.+\frac{3 \mathfrak{q}^{4}-18 \mathfrak{w}^{2} \mathfrak{q}^{2}+7 \mathfrak{w}^{4}}{4\left(\mathfrak{q}^{2}-\mathfrak{w}^{2}\right)^{2}}\left(H_{a a}^{0}\right)^{2}-\frac{3 \mathfrak{q}^{4}+14 \mathfrak{w}^{2} \mathfrak{q}^{2}-9 \mathfrak{w}^{4}}{\left(\mathfrak{q}^{2}-\mathfrak{w}^{2}\right)^{2}}\left(H_{t z}^{0}\right)^{2}\right]
\end{aligned}
$$


Equation (A.6) appears to contain more than just contact terms, since there is a pole at $|\mathfrak{w}|=\mathfrak{q}$. This pole, however, is artificial - it reflects the normalization of $Z_{2}$, and cancels in the final expression for the correlators.

If one chooses to keep track of contact terms in field theory correlators, one can use $S_{C T}^{(2)}$ to reproduce contact terms computed earlier in [16]. For example,

$$
G_{t t, t t}=\frac{2 q^{4}}{3\left(q^{2}-\omega^{2}\right)^{2}} G_{2}(\omega, q)-4 \frac{\delta^{2} S_{C T}^{(2)}(0)}{\left(\delta H_{t t}^{0}\right)^{2}}
$$

in the hydrodynamic limit becomes

$$
G_{t t, t t}=\frac{3 N_{c}^{2} \pi^{2} T^{4}\left(3 \omega^{2}-5 q^{2}\right)}{8\left(q^{2}-3 \omega^{2}\right)}
$$

which coincides with the result of [16].

\section{B. Frobenius solution}

To find the full quasinormal spectrum, one has to analyze wave equations (4.5), (4.18), (4.26), and (4.35) in more detail. All these equations are Fuchsian ODEs with $k$ singular points, ${ }^{19}$ two of which correspond, respectively, to the horizon $(u=1)$ and the boundary $(u=0)$. For all of the above equations, the exponents at the horizon are equal to $\pm i \mathfrak{w} / 2$, corresponding to two local solutions representing outgoing (incoming) waves. The solution obeying incoming wave boundary condition at the horizon can be represented as a power series around $u=1$,

$$
Z(u)=(1-u)^{-i \mathfrak{w} / 2}(1+u)^{-\mathfrak{w} / 2} \sum_{n=0}^{\infty} a_{n}(\mathfrak{w}, \mathfrak{q})(1-u)^{n},
$$

where $Z(u)$ stands for either of $E_{\alpha}, E_{z}, Z_{1}, Z_{2}, Z_{3}$. The coefficients $a_{n}$ of the series expansion obey $(k-1)$-term recursion relations which can be found by substituting (B.1) in the original differential equations. Quasinormal spectrum is determined by imposing Dirichlet boundary condition at $u=0$,

$$
Z(0)=\sum_{n=0}^{\infty} a_{n}(\mathfrak{w}, \mathfrak{q})=0
$$

and solving Eq. (B.2) numerically taking a sufficiently large but finite number of terms in the sum. ${ }^{20}$ The spectra of all of the above wave equations are qualitatively similar, except for the

\footnotetext{
${ }^{19}$ For the transverse Maxwell equation (4.5a) satisfied by $E_{\alpha}$, and for the scalar channel wave equation (4.18) satisfied by $Z_{3}$, the number of singular points is $k=4$, corresponding to $u=0, \pm 1, \infty$. For the longitudinal Maxwell equation (4.5b) satisfied by $E_{z}$, and the shear channel wave equation (4.26) satisfied by $Z_{1}$, the number of singular points is $k=6$, corresponding to $u=0, \pm 1, \pm \sqrt{1-\mathfrak{w}^{2} / \mathfrak{q}^{2}}, \infty$. For the sound channel wave equation (4.35) satisfied by $Z_{3}$, the number of singular points is also $k=6$, corresponding to $u=0, \pm 1, \pm \sqrt{3\left(1-\mathfrak{w}^{2} / \mathfrak{q}^{2}\right)}, \infty$.

${ }^{20}$ Careful readers may note that the expansion (B.1) is guaranteed to converge only inside a circle of radius $\rho$ around $u=1$ (in the complex $u$ plane), where $\rho$ is the distance to the nearest singular point, which may become less than one (distance to the boundary) for some values of $\mathfrak{w}$ and $\mathfrak{q}$. However, even for such values of $\mathfrak{w}$ and $\mathfrak{q}$, our numerical results for diffusive and shear modes are in agreement with previous calculations [11] where this issue did not arise, thus suggesting wider applicability of the expansion (B.1).
} 

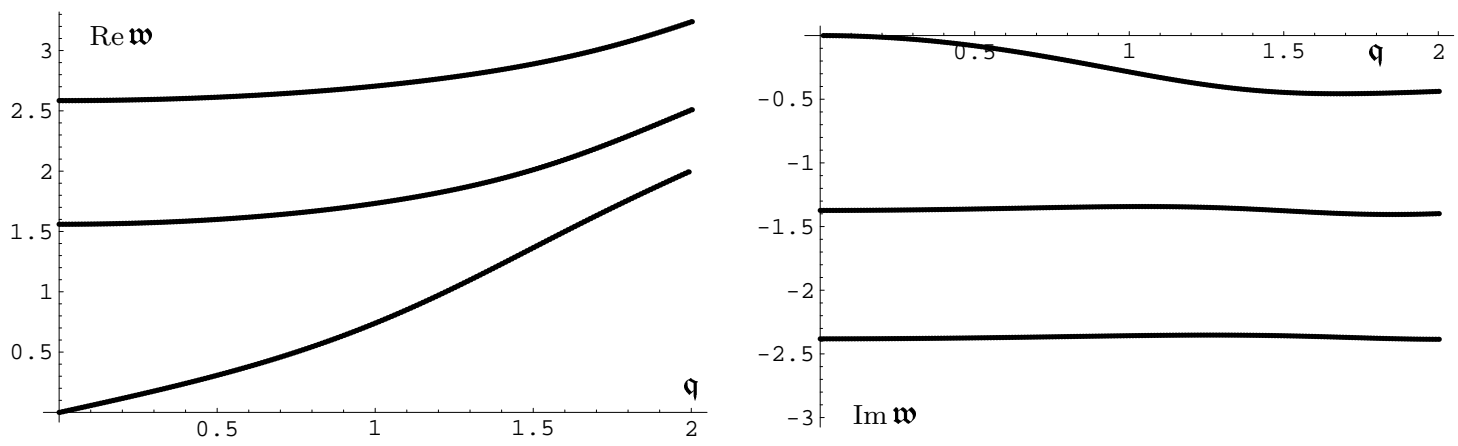

Figure 6: Real and imaginary parts of three lowest quasinormal frequencies as function of spatial momentum. The curves for which $\mathfrak{w} \rightarrow 0$ as $\mathfrak{q} \rightarrow 0$ correspond to hydrodynamic sound mode in the dual finite temperature $\mathcal{N}=4$ SYM theory.

behavior of the lowest (hydrodynamic) frequency which is absent for $E_{\alpha}$ and $Z_{3}$. For $E_{z}$ and $Z_{1}$, hydrodynamic frequencies are purely imaginary (given by Eqs. (4.16) and (4.32) for small $\omega$ and $q$ ), and presumably move off to infinity as $q$ becomes large. For $Z_{2}$, the hydrodynamic frequency has both real and imaginary parts (given by Eq. (4.44) for small $\omega$ and $q$ ), and eventually (for large $q$ ) becomes indistinguishable in the tower of other eigenfrequencies. As an example, dispersion relations for the three lowest quasinormal frequencies in the sound channel (including the one of the sound wave) are shown in Fig. 6. The tables below give numerical values of quasinormal frequencies for $\mathfrak{q}=1$. Only non-hydrodynamic frequencies are shown in the tables. The position of hydrodynamic frequencies at $\mathfrak{q}=1$ is $\mathfrak{w}=-3.250637 i$ for the $R$-charge diffusive mode, $\mathfrak{w}=-0.598066 i$ for the shear mode, and $\mathfrak{w}= \pm 0.741420-0.286280 i$ for the sound mode. The numerical values of the lowest five (non-hydrodynamic) quasinormal frequencies for electromagnetic perturbations are:

\begin{tabular}{|c||c|c||c|c|}
\hline \multicolumn{1}{|c||}{} & \multicolumn{2}{c||}{ Transverse channel } & \multicolumn{2}{c|}{ Diffusive channel } \\
\cline { 2 - 5 }$n$ & $\operatorname{Re} \mathfrak{w}$ & $\operatorname{Im} \mathfrak{w}$ & $\operatorname{Re} \mathfrak{w}$ & $\operatorname{Im} \mathfrak{w}$ \\
\hline 1 & \pm 1.547187 & -0.849723 & \pm 1.147831 & -0.559204 \\
2 & \pm 2.398903 & -1.874343 & \pm 1.910006 & -1.758065 \\
3 & \pm 3.323229 & -2.894901 & \pm 2.903293 & -2.891681 \\
4 & \pm 4.276431 & -3.909583 & \pm 3.928555 & -3.943386 \\
5 & \pm 5.244062 & -4.920336 & \pm 4.946818 & -4.965186 \\
\hline
\end{tabular}

and for gravitational perturbations are:

\begin{tabular}{|c||c|c||c|c||c|c|}
\hline \multicolumn{1}{|c||}{} & \multicolumn{2}{c||}{ Scalar channel } & \multicolumn{2}{c||}{ Shear channel } & \multicolumn{2}{c|}{ Sound channel } \\
\cline { 2 - 7 }$n$ & $\operatorname{Re} \mathfrak{w}$ & $\operatorname{Im} \mathfrak{w}$ & $\operatorname{Re} \mathfrak{w}$ & $\operatorname{Im} \mathfrak{w}$ & $\operatorname{Re} \mathfrak{w}$ & $\operatorname{Im} \mathfrak{w}$ \\
\hline 1 & \pm 1.954331 & -1.267327 & \pm 1.759116 & -1.291594 & \pm 1.733511 & -1.343008 \\
2 & \pm 2.880263 & -2.297957 & \pm 2.733081 & -2.330405 & \pm 2.705540 & -2.357062 \\
3 & \pm 3.836632 & -3.314907 & \pm 3.715933 & -3.345343 & \pm 3.689392 & -3.363863 \\
4 & \pm 4.807392 & -4.325871 & \pm 4.703643 & -4.353487 & \pm 4.678736 & -4.367981 \\
5 & \pm 5.786182 & -5.333622 & \pm 5.694472 & -5.358205 & \pm 5.671091 & -5.370784 \\
\hline
\end{tabular}




\section{References}

[1] J. D. Bekenstein, "Black Holes And Entropy," Phys. Rev. D 7, 2333 (1973); J. M. Bardeen, B. Carter and S. W. Hawking, "The Four Laws Of Black Hole Mechanics," Commun. Math. Phys. 31, 161 (1973); S. W. Hawking, "Particle Creation By Black Holes," Commun. Math. Phys. 43, 199 (1975).

[2] J. M. Maldacena, "The large $N$ limit of superconformal field theories and supergravity," Adv. Theor. Math. Phys. 2, 231 (1998) [Int. J. Theor. Phys. 38, 1113 (1999)], hep-th/9711200.

[3] S. S. Gubser, I. R. Klebanov and A. M. Polyakov, "Gauge theory correlators from non-critical string theory," Phys. Lett. B 428, 105 (1998), hep-th/9802109.

[4] E. Witten, "Anti-de Sitter space and holography," Adv. Theor. Math. Phys. 2, 253 (1998), hep-th/9802150.

[5] O. Aharony, S. S. Gubser, J. M. Maldacena, H. Ooguri and Y. Oz, "Large N field theories, string theory and gravity," Phys. Rept. 323, 183 (2000), hep-th/9905111.

[6] K. D. Kokkotas and B. G. Schmidt, "Quasi-normal modes of stars and black holes," Living Rev. Rel. 2, 2 (1999), gr-qc/9909058; H. P.Nollert, "Quasinormal modes: the characteristic "sound" of black holes and neutron stars," Class. Quantum Grav. 16, R159 (1999); I. Sachs, "Quasi normal modes," Fortsch. Phys. 52, 667 (2004), hep-th/0312287.

[7] G. T. Horowitz and V. E. Hubeny, "Quasinormal modes of AdS black holes and the approach to thermal equilibrium," Phys. Rev. D 62, 024027 (2000), hep-th/9909056.

[8] D. Birmingham, I. Sachs and S. N. Solodukhin, "Conformal field theory interpretation of black hole quasi-normal modes," Phys. Rev. Lett. 88, 151301 (2002), hep-th/0112055.

[9] D. T. Son and A. O. Starinets, "Minkowski-space correlators in AdS/CFT correspondence: Recipe and applications," JHEP 0209, 042 (2002), hep-th/0205051.

[10] I. G. Moss and J. P. Norman, "Gravitational quasinormal modes for anti-de Sitter black holes," Class. Quant. Grav. 19, 2323 (2002), gr-qc/0201016.

[11] A. Nunez and A. O. Starinets, "AdS/CFT correspondence, quasinormal modes, and thermal correlators in $N=4$ SYM," Phys. Rev. D 67, 124013 (2003), hep-th/0302026.

[12] R. A. Konoplya, "Gravitational quasinormal radiation of higher-dimensional black holes," Phys. Rev. D 68, 124017 (2003), hep-th/0309030.

[13] J. M. Bardeen, “Gauge invariant cosmological perturbations," Phys. Rev. D 22 (1980) 1882.

[14] G. Policastro, D. T. Son and A. O. Starinets, "The shear viscosity of strongly coupled $N=4$ supersymmetric Yang-Mills plasma," Phys. Rev. Lett. 87, 081601 (2001), hep-th/0104066.

[15] G. Policastro, D. T. Son and A. O. Starinets, "From AdS/CFT correspondence to hydrodynamics," JHEP 0209, 043 (2002), hep-th/0205052.

[16] G. Policastro, D. T. Son and A. O. Starinets, "From AdS/CFT correspondence to hydrodynamics. II: Sound waves," JHEP 0212, 054 (2002), hep-th/0210220.

[17] D. Forster, Hydrodynamic Fluctuations, Broken Symmetry, and Correlation Functions, Benjamin/Cummings, 1975. 
[18] E. L. Ince, Ordinary differential equations, Dover, New York, 1956.

[19] I. R. Klebanov and E. Witten, "AdS/CFT correspondence and symmetry breaking," Nucl. Phys. B 556, 89 (1999), hep-th/9905104.

[20] C. P. Herzog and D. T. Son, "Schwinger-Keldysh propagators from AdS/CFT correspondence," JHEP 0303, 046 (2003), hep-th/0212072.

[21] V. I. Arnol'd, Ordinary Differential Equations, Springer-Verlag, New York, 1992.

[22] U. H. Danielsson, E. Keski-Vakkuri and M. Kruczenski, "Spherically collapsing matter in AdS, holography, and shellons," Nucl. Phys. B 563, 279 (1999), hep-th/9905227.

[23] P. Kovtun, D. T. Son and A. O. Starinets, "Viscosity in strongly interacting quantum field theories from black hole physics," Phys. Rev. Lett. 94, 111601 (2005), hep-th/0405231.

[24] D. Z. Freedman, S. D. Mathur, A. Matusis and L. Rastelli, "Correlation functions in the $C F T(d) / A d S(d+1)$ correspondence," Nucl. Phys. B 546, 96 (1999), hep-th/9804058.

[25] G. Chalmers, H. Nastase, K. Schalm and R. Siebelink, "R-current correlators in $N=4$ super Yang-Mills theory from anti-de Sitter supergravity,” Nucl. Phys. B 540, 247 (1999), hep-th/9805105.

[26] A. O. Starinets, "Quasinormal modes of near extremal black branes," Phys. Rev. D 66, 124013 (2002), hep-th/0207133.

[27] J. Natario and R. Schiappa, "On the classification of asymptotic quasinormal frequencies for d-dimensional black holes and quantum gravity," hep-th/0411267.

[28] P. Kovtun and L. G. Yaffe, "Hydrodynamic fluctuations, long-time tails, and supersymmetry," Phys. Rev. D 68, 025007 (2003), hep-th/0303010.

[29] E. Witten, "Baryons in the 1/N expansion," Nucl. Phys. B 160, 57 (1979). 